HIAS-E-64

\title{
Radial Basis Functions Neural Networks for Nonlinear Time Series Analysis and Time-Varying Effects of Supply Shocks
}

\author{
Nobuyuki Kanazawa
}

Hitotsubashi Institute for Advanced Study, Hitotsubashi University

March 2018

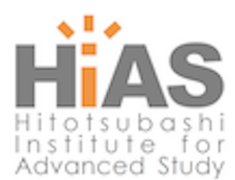

Hitotsubashi Institute for Advanced Study, Hitotsubashi University

2-1, Naka, Kunitachi, Tokyo 186-8601, Japan

tel:+81 425808604 http://hias.ad.hit-u.ac.jp/

HIAS discussion papers can be downloaded without charge from: http://hdl.handle.net/10086/27202

https://ideas.repec.org/s/hit/hiasdp.html

All rights reserved. 


\title{
Radial Basis Functions Neural Networks for Nonlinear Time Series Analysis and Time-Varying Effects of Supply Shocks
}

\author{
Nobuyuki Kanazawa*
}

March 8, 2018

\begin{abstract}
I propose a flexible nonlinear method for studying the time series properties of macroeconomic variables. In particular, I focus on a class of Artificial Neural Networks (ANN) called the Radial Basis Functions (RBF). To assess the validity of the RBF approach in the macroeconomic time series analysis, I conduct a Monte Carlo experiment using the data generated from a nonlinear New Keynesian (NK) model. I find that the RBF estimator can uncover the structure of the nonlinear NK model from the simulated data whose length is as small as 300 periods. Finally, I apply the RBF estimator to the quarterly US data and show that the response of the macroeconomic variables to a positive supply shock exhibits a substantial time variation. In particular, the positive supply shocks are found to have significantly weaker expansionary effects during the zero lower bound periods as well as periods between 2003 and 2004. The finding is consistent with a basic NK model, which predicts that the higher real interest rate due to the monetary policy inaction weakens the effects of supply shocks.
\end{abstract}

Keywords: Neural Networks; Radial Basis Functions; Zero Lower Bound; Supply Shocks.

JEL Classification: C45; E31.

${ }^{*}$ Hitotsubashi Institute for Advanced Study (HIAS), Hitotsubashi University (email: n.kanazawa@r.hit-u.ac.jp): I would especially like to thank Karel Mertens, Levon Barseghyan, and Christopher Huckfeldt for their great guidance and support. All remaining errors are mine. 


\section{Introduction}

In this paper, I propose an Artificial Neural Network (ANN)-based nonlinear VAR estimator with a particular focus on the class of network called the Radial Basis Functions (RBF). The use of RBF is motivated by its ability to flexibly estimate a complex function. Despite its flexibility, however, the RBF can be efficiently estimated as if it is a linear estimator by following a simple two-step estimation procedure that is commonly adopted in the neural network community.

The RBF has been studied extensively in the field of computer science and neural networks, and it has been shown that the RBF can approximate any continuous functions on a compact domain (known as the Universal Approximation Property). ${ }^{1}$ Unlike typical regime-switching VARs, the network-based model can flexibly estimate a potentially state-dependent and nonlinear data-generating process as a whole, making it possible to produce nonlinear impulse responses without specifying potentially large and unknown number of state. This feature is especially useful when econometricians do not have a clear understanding of the underlying data-generating process. The strengths of the RBF time series are similar to the models that admit time variation in multivariate linear structures or the Time-Varying Coefficient VARs (TVCVAR). ${ }^{2}$ Both RBF and TVC-VAR estimators are capable of capturing very general nonlinear dynamics without specifying the state of an economy. An additional benefit of the RBF estimator, however, is its low computational costs. As pointed out by Kilian and Lütkepohl (2017), the Bayesian estimation of the TVC-VAR is only possible, in practice, for small dimensional models with a small number of lags due to its high computational costs. The curse of dimensionality is greatly reduced for the RBF estimator because its estimation is conducted by the two-step procedure that essentially breaks down a costly nonlinear estimation into (1) a fast clustering method and (2) a linear estimation. ${ }^{3}$ The benefit of low computational costs allows researchers to estimate nonlinear models that can produce time-varying impulse response functions with a number of dimension as large as what is typically used in a linear VAR estimation.

In order to validate the use of the RBF estimator in the macroeconomic analysis,

\footnotetext{
${ }^{1}$ See Hartman et al. (1990) and Park and Sandberg (1991).

${ }^{2}$ Examples include Primiceri (2005), Canova (1993), Sims (1993), Stock and Watson (1996) and Cogley and Sargent (2001)

${ }^{3}$ This procedure is called the 'unsupervised learning' in the neural nets field.
} 
one needs to test if the estimator can correctly capture the structure of aggregate economy with the number of observations that is usually available to a macroeconomist. This is because, despite the universal approximation property of the RBF, the rate of convergence generally depends on the smoothness of the function to be approximated and the number of available observations. To this end, I conduct a Monte-Carlo experiment using the simulated time series data generated from a medium-scale, nonlinear New Keynesian (NK) model, which is frequently viewed as a representation of aggregate economy among economists and policy makers. Nonlinearity in this NK model comes from a kink in the central bank's monetary policy rule where the interest rates is bounded below at $0 \%$. I then calculate the within-sample Mean-SquaredErrors (MSEs) as defined by the distance between the true impulse responses from the model and the impulse responses estimated by the RBF estimator. For comparison, I also conduct the same exercise using the linear VARs. I found that the RBF estimator outperforms the linear VARs even when the sample size is limited to as small as 300 periods. The result suggests that the RBF time series can correctly capture the nonlinear structure of the medium-scale NK model and that the use of the RBF estimator for macroeconomic time series analysis may be appropriate.

Finally, I apply the RBF estimator to the US quarterly data from 1978 to 2016. Using Fernald (2014)'s utilization-adjusted TFP shocks, I estimate impulse responses of the output, consumption, investment, hours worked, expected inflation rate, and nominal interest rate. The aim of this exercise is to test the prediction of the textbook NK model, which states that the expansionary effects of a positive supply shock is weaker under the passive monetary policy regimes such as the zero lower bound (zlb) periods. Consistent with the NK model's prediction, I found that the effects of the positive TFP shock is significantly weaker during the periods of zlb.

Furthermore, the output responses to the supply shocks are found to be similarly small between 2003 and 2004. During these periods, the federal funds rate was very low at around 1\%. In fact, the estimated nominal interest rate response suggests that the Fed was not responding actively to the supply shocks during these periods. As a consequence of the unresponsive nominal interest rate and the deflationary effects, the supply shocks are estimated to raise the real interest rate during these periods, which discourages consumption, investment, and output. The finding highlights the critical role that the response of real interest rate plays in shaping the responses of other macroeconomic variables. The study also suggests that the estimated responses of macroeconomic variables around 2003-2004 is remarkably similar to the responses 
during the zlb periods around 2008-2015.

These results are in contrast with the existing studies (Garín et al. (2016) and Wieland (2016)) that found the strong expansionary effects of a positive supply shock during the periods of zlb. I briefly discuss potential explanations for the difference in the findings between the current paper and the existing studies and show that it is likely to be a consequence of different data specifications rather than the different methodologies. Specifically, I estimated the output response using the statedependent local projection method and show that the effects of the supply shocks are significantly weaker during the zlb periods once the growth rate specifications is used and an outlier (2008Q4) is excluded. The exercise reiterates the point that the macroeconomic dynamics during the passive monetary policy regimes are markedly different from the usual macroeconomic dynamics under the active monetary policy regimes as prescribed by the basic NK model.

The rest of the paper is organized as follows: Section 2 reviews literature on the Radial Basis Functions. Section 3 goes over literature on time series analysis. Section 4 describes the Monte Carlo simulation exercise, Section 5 illustrates empirical application to the U.S. data, Section 6 briefly discusses the results, and Section 7 concludes.

\section{Radial Basis Functions}

In this paper, I focus on a particular class of the ANN called the Radial Basis Functions. The ANN typically consists of several layers between input and output variables. Each layer is made of multiple units, and a unit in one layer receives inputs from the preceding layers and produces outputs for the succeeding layer. The RBF consists of three layers with a single hidden layer between the input and output layers. ${ }^{4}$ Figure 1 shows the graphical description of the RBF. The three layers depicted in the figure are: (1) an input layer, where the independent variables enter the system; (2) a hidden layer, where the independent variables are transformed; and (3) a linear output layer, where the dependent variables are predicted.

The RBF was first introduced as a solution technique for interpolation problems, and in the late 1980s, the RBF formulation was extended to perform more general

\footnotetext{
${ }^{4}$ The word "hidden" is used to distinguish the layer in the middle from the input and output layers
} 


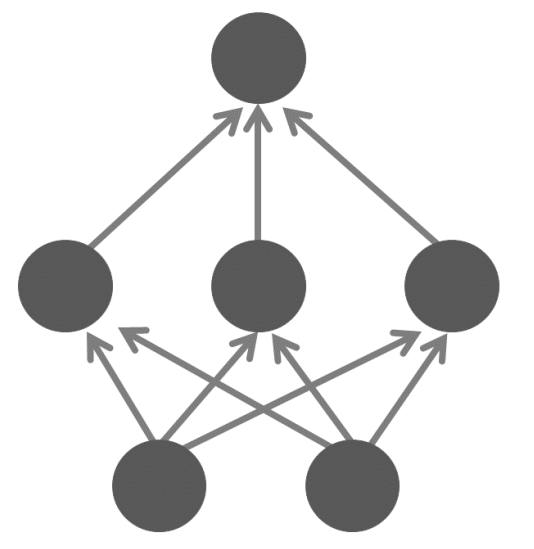

\section{Output y}

\section{Linear weights}

\section{Hidden layer}

\section{Weights}

Input $\mathbf{x}$

Figure 1: Graphical description of RBF

task of approximation. ${ }^{5}$ Since then, a number of researchers including Park and Sandberg (1991), Xu et al. (1994), and Girosi and Poggio (1990) showed that any continuous function on a compact domain can be approximated arbitrarily well by the RBF, making the RBF one of the popular choices among various ANNs.

Consider some unknown function $y=f(x)$, which is to be approximated given a dataset $\left(x_{t}, y_{t}\right) \cdot f(\cdot)$ is a nonlinear function or the conditional expectation of $y_{t}$ given $x_{t}$, hence:

$$
y_{t}=f\left(x_{t}\right)+\epsilon_{t}, \quad E\left[\epsilon_{t} \mid x_{t}\right]=0
$$

In the case of the RBF, $f\left(x_{t}\right)$ takes the following functional form:

\footnotetext{
${ }^{5}$ See Broomhead and Lowe (1988), Moody and Darken (1989), and Poggio and Girosi (1990).
} 


$$
\begin{aligned}
f\left(x_{t}\right) & =\sum_{j=1}^{M} K_{\lambda}\left(\xi_{j}, x_{t}\right) \beta_{j} \\
& =\sum_{j=1}^{M} D\left(\frac{\left\|x_{t}-\xi_{j}\right\|}{\lambda}\right) \beta_{j}
\end{aligned}
$$

where each basis element is indexed by a location parameter or a centroid, $\xi_{j}$, and a scale parameter $\lambda . \quad M$ is the number of centroids in the model, and $\lambda$ has the same number of dimension as the input, $x$. I choose the gaussian density function for the kernel, $D$, since it is the most popular choice of the base function for the RBF. Roughly speaking, when a new input enters the system, the RBF computes the Euclidean distance between the input and each of the centroids, $\xi_{j}$, and assigns densities using the gaussian density function. The RBF then computes the predicted value, $\widehat{y}_{t}$, based on linear combinations of the densities and the weights, which is given by $\beta_{j}$. I estimate the three sets of parameters, $\xi_{j}, \lambda$, and $\beta_{j}$ by solving the following minimization problem:

$$
\min _{\left\{\lambda, \xi_{j}, \beta_{j}\right\}_{1}^{M}} \sum_{t=1}^{T}\left(y_{t}-\beta_{0}-\sum_{j=1}^{M} \beta_{j} \exp \left\{-\frac{\left(x_{t}-\xi_{j}\right)^{\prime}\left(x_{t}-\xi_{j}\right)}{\lambda^{2}}\right\}\right)^{2}
$$

The weights between the input layer and the hidden layer in Figure 1 is represented as $\lambda^{\prime} s$, the units in the hidden layer are represented by $\xi_{j}$, and the linear weights between the hidden layer and the output layer is represented as $\beta^{\prime} s$ in the equation 3. The problem looks complex and highly nonlinear at first. However, I show in Section 2.1 that this minimization problem can be solved quickly as if it is a linear optimization problem.

Extending the RBF formulation to a lag-dependent model is straightforward by augmenting the RBF model with lags, denoted by $p$. Thus, the lag-dependent RBF model is as follows:

$$
y_{t}=\sum_{j=1}^{M} K_{\lambda}\left(\xi_{j},\left\{y_{t-p}\right\}_{p=1}^{P}\right) \beta_{j}+\epsilon_{t}
$$

where $\epsilon_{t}$ is a vector of error terms, and the independent variables are now the lagged dependent variables. 


\subsection{Parameter Estimation Methods and Renormalization}

As specified in Section 2, there are three types of parameters to estimate: centroids $\xi_{j}$, scale parameters $\lambda$, and weights $\beta_{j}$. Rate of convergence and accuracy of solution generally depend on the method of estimation. Since this paper is not intended for finding the optimal method of estimation, I simply choose one of the popular estimation methods that breaks down the complex nonlinear minimization problem into two simple steps. ${ }^{6}$ In other words, the parameters in the hidden layer and the weights $(\beta)$ are estimated separately. Some argue that the global solution $\operatorname{method}^{7}$ is ideal in order to ensure that the equation 2 is globally minimized. However, such a method exponentially increases the computational costs, and, consequently, the global solution method is rarely used in practice. The two-step estimation procedure is as follows. First, I employ a clustering method to fix the value of centroids, $\xi_{j}$. A common choice is the $\mathrm{K}$-means clustering method, in which $n$ observations are assigned into $k$ clusters with each observation belonging to the cluster with nearest mean. The "mean" of each cluster constitutes a prototype of that cluster, and for this reason, I set the centroids to be the "means" of the clusters. Figure 2 illustrates the Kmeans clustering method. In this illustration, 1000 observations are partitioned into four clusters, with yellow stars representing the "means" of each cluster. Essentially, the RBF estimator constructs kernels (the gaussian density functions) centering these centriods, and, therefore, the centroids need to be a good representation of the entire sample so that the RBF kernels have a comprehensive coverage over the sample.

In general, centroids determined by the K-means clustering are not unique, and the location of centriods depends on the initial values. For this reason, I conduct a primitive, random search optimization by simply repeating the K-means clustering algorithm with 1,000 different initial values and select centroids that minimize the error. Other centroid-selection methods were also considered including the Orthogonal Least Square Algorithm by Chen et al. (1991). However, the crude optimization of the random search over different initial values repeatedly produced lower error and was more stable.

In addition to determining the centroids, the scale parameters, $\lambda$, need to be estimated as well. I fix $\lambda$ to be the standard deviation of $x$ so that the kernel of

\footnotetext{
${ }^{6}$ This method of training is also called the "unsupervised training,"

${ }^{7}$ Or, in the language of neural networks literature, "supervised training," in which the backpropagation is activated
} 
Figure 2: Illustration of K-means clustering

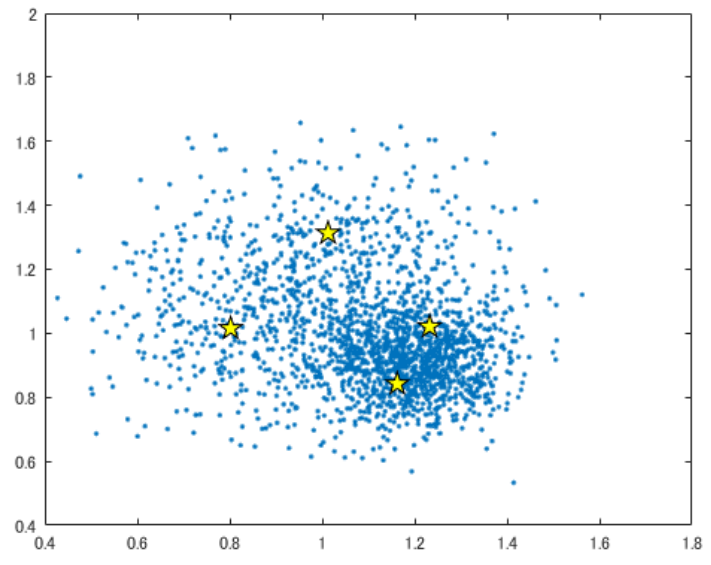

(a) Observations and "Means"

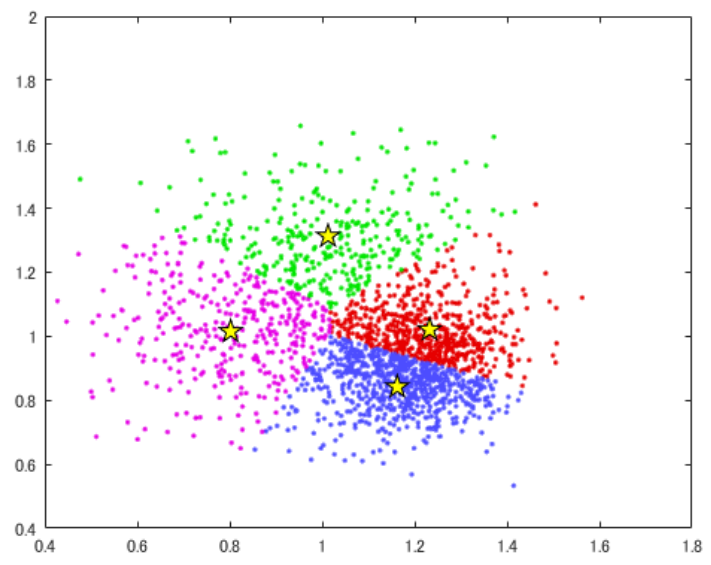

(b) Clusters

the RBF in equation (2) is akin to the standard normal density function. Fixing $\lambda$ is convienient for computational efficiency, but it has a side-effect of leaving holeregions in the input space where no kernel has appreciable support. For illustration, the hole-regions as well as the support from the RBF kernels are depicted in the top panel of Figure 3. With kernel's width being fixed, there is a region where no kernel support can be reached. To avoid this problem, Friedman et al. (2008) suggested to use the normalized RBF, which is specified as follows:

$$
y=\sum_{j=1}^{M} h_{j}(x) \beta_{j}+\epsilon
$$

where

$$
h_{j}(x)=\frac{D\left(\left\|x-\xi_{j}\right\|\right)}{\sum_{k=1}^{M} D\left(\left\|x-\xi_{k}\right\|\right)}
$$

The bottom panel of Figure 3 illustrates the kernel support using the normalized $\mathrm{RBF}$, which is now extended to cover the entire region. I use the normalized RBF in the remainder of the paper.

A convenient property of the RBF is that once $\xi_{j}$ and $\lambda$ are fixed, the function is linear in $\beta$ 's. Thus, $\beta_{j}$ can be estimated in the usual OLS manner. I choose the 

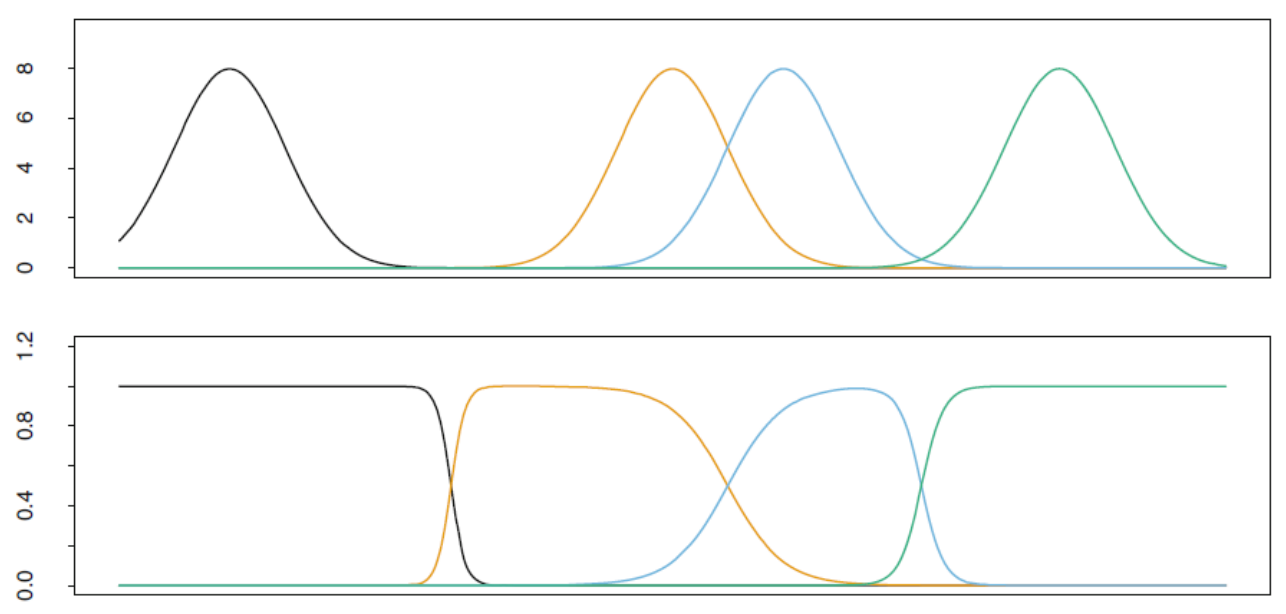

Figure 3: Top panel: Radial Basis Functions. Bottom panel: Nomalized RBF (Reprinted from Friedman et al. (2008)).

targeted number of centroids, $M$, by the Bayesian Information Criteria (BIC). ${ }^{8}$

\section{Literature Review}

This paper is related to the literature on nonlinear time series analysis. The literature can be generally classified into three.

The first approach to capture nonlinearities relies on the use of regime-switching VAR models including the threshold VARs (e.g., Hubrich and Teräsvirta (2013)) and the Markov-switching VARs (Hamilton (1989)). One caveat of this approach is that, while regime-switching VARs can capture state dependence of macroeconomic variables, it assumes that the economy can be described by a finite number of regimes, within which the structures of the economy are assumed to be the same. This approach becomes computationally challenging as the number of regimes increases or if the true data generating process features asymmetric impulse responses within each state. In such a case, a new set of VAR coefficients need to be estimated for each new regime. In contrast, the RBF estimator can efficiently estimate a possibly state-

\footnotetext{
${ }^{8}$ I also compute the Akaike Information Criteria, but BIC gives a clearer indication of the optimal number of centroids.
} 
dependent and asymmetric structure of the economy as a whole without actually specifying the state or the structure of the economy.

The second approach consists of directly estimating the impulse response functions. This strand of approach was pioneered by Jordà (2005) who proposed the Local Projection method. While Jorda's method can easily capture nonlinearities in the response functions, his method poses serious difficulty when its efficiency is considered as pointed out by Barnichon and Matthes (2016). Indeed, drawing inferences on a rich set of nonlinearities including sign- and state-dependence using the local projection method is often difficult.

Relatedly, new approach is recently proposed by Barnichon and Matthes (2016) who uses few numbers of gaussian functions to approximate the impulse functions. Like Jordà (2005), this approach is robust to a functional-form assumtion error but has a great advantage of reduced efficiency costs because, instead of model-free estimation of Jordà (2005), Barnichon and Matthes (2016) imposes a flexible parametric assumption based on the mixture of gaussian base functions. The current paper is similar to Barnichon and Matthes (2016)'s in that both papers utilize the gaussian functions to approximate macroeconomic dynamics. However, our papers are different in the sense that the current paper employs the neural network structure that makes use of the hidden layer. This multiple-layer structure of the RBF provides extra flexibility, which makes it possible to estimate a nonlinear VAR system without specifying the state of an economy. In contrast, estimating asymmetric or state-dependent impulse responses using Barnichon and Matthes (2016)'s method still requires researchers to specify the states of the economy often in the form of indicator variables. Thus, the RBF method can be an useful alternative when there is little information about the states or the structure of the true data-generating process. At the same time, one caveat for using the RBF estimator is that researchers need to assume the existence of a VAR representation in the form of the equation 4 . This assumption is not neccesary for Barnichon and Matthes (2016) and Jordà (2005) because they directly estimate the impulse response functions.

The third approach is the TVC-VAR models (e.g. Primiceri (2005)). As suggested by Kilian and Lütkepohl (2017), even though the TVC-VAR can capture very general nonlinear dynamics without specifying the states of an economy, its expensive computational cost often prohibits researchers from estimating large dimensional models and, thus, limiting the use of TVC-VAR in practice. Unlike the TVC-VAR, the RBF 
estimator can be estimated with a relatively low computational cost. Therefore, estimation of a larger dimensional model with a larger number of autoregressive lags can be relatively easily handled by the RBF. ${ }^{9}$

In addition to the nonlinear time series literature listed above, the current paper is obviously related to the literature that employs the ANN in economic and financial time series analysis. The majority of the research that applied the ANN in economics so far has been focused on forecasting the financial variables. In those studies, the ANN models are often found to outperform the traditional time series models. ${ }^{10}$. Few studies tested the ANN's ability to forecast macroeconomic variables. Swanson and White (1997) applied ANN models to forecast nine US macroeconomic series and concluded that even though their results were mixed, the ANN models were promising even when no explicit nonlinearity was found in the macroeconomic dynamics. Moshiri and Cameron (1999) investigated if the ANN can correctly forecast inflation and Tkacz (2001) used the ANN for forecasting the Canadian GDP. An extensive review of the use of the ANN in the context of economic analysis was written by Kuan and White (1994).

\section{Monte Carlo simulation}

To study the validity of the RBF estimator in the macroeconomic time series analysis, I conduct the Monte Carlo simulation in this section. I ask: "if the true datagenerating process of an economy was a medium-sized nonlinear New Keynesian (NK) model, can the RBF networks uncover the structure of the NK model from the simulated dataset?" To answer this question, I first solve a nonlinear NK model globally, and simulate data using this NK model. The nonlinearity in this NK model stems from the kink in the central bank's Taylor rule, in which the central bank cannot lower the interest rate below zero. Except for the habit persistence in the household sector, the NK model is standard. In Appendix 8.1, I describe the NK model used for simulation in detail. After I solved the model, I simulated time series data for 1 million periods. The nominal interest rate hits zero for about $1.85 \%$ of the simulated periods.

\footnotetext{
${ }^{9}$ In comparing with the TVC-VAR, one limitation of the RBF estimator is that it does not admit time-varying variance covariance matrix.

${ }^{10}$ See Hutchinson et al. (1994), Lachtermacher and Fuller (1995), Zhang and Wan (2007), Zhang and $\mathrm{Hu}$ (1998), and Guresen et al. (2011)
} 


\subsection{Mean Squared Error Comparison}

Using the time series data generated by the NK model, I calculate the within-sample MSEs of the impulse responses estimated by the RBF estimator and linear VARs. The MSEs are measured by the deviation of the estimated impulse responses from the true impulse responses. I present the MSEs under two different scenarios. In the first scenario, the economy is in the "normal" state, in which the nominal interest rates is strictly positive. I make sure that the economy is far away from the zlb state by ensuring that the nominal interest rates is greater than $1 \%$ for at least five consecutive periods prior to and after the shock hits the economy. The second scenario considers the economy under the "zlb" state, in which the nominal interest rate is binding at zero. To ensure that the economy is distinctively different from the normal state, I define the zlb state as the periods where the nominal interest rate is zero for at least three consecutive periods. When calculating the MSEs, I produce the impulse responses by giving the shock to the second of the three consecutive zlb periods.

The exogenous state variable for the NK economy is the productivity shock, $a_{t}$, and the endogenous state variables are (1) price dispersion, (2) consumption, and (3) interest rates. Since econometricians typically do not have a good measure of price dispersion, I instead assume that I observe the inflation rate instead of the price dispersion.

Let $X_{t}=\left[\pi_{t+1}, C_{t}, R_{t}\right]$. Our RBF estimator can be written as follows:

$$
X_{t}=R B F\left(a_{t},\left\{a_{t-p}, X_{t-p}\right\}_{p=1}^{P}\right)+\epsilon_{t}
$$

Since $a_{t}$ is orthogonal to the other variables, I impose the assumption that the productivity shock can affect the macroeconomic variables contemporaneously, but not vice versa. I impose the same assumption when I estimate the VAR model by ordering $a_{t}$ at the very first. ${ }^{11}$

One complication arises as I estimate nonlinear impulse response functions, which generally depend on future shocks and current state of the economy (history) as well as the sign and magnitude of the current shock. To accomodate the impulse responses to various future shocks, I use the generalized impulse response functions following

\footnotetext{
${ }^{11} \mathrm{I}$ also added the measurement error to the simulated inflation. The error follows the normal distribution with standard deviation equal to the $3 \%$ of the standard deviation of $\pi$.
} 
Koop et al. (1996). ${ }^{12}$ Let GI denote a generalized impulse response function. Then, the impulse response of a variable, $Y_{t+n}$, in $n$-period ahead is:

$$
G I\left(n, v_{t}, \omega_{t-1}\right)=E\left[Y_{t+n} \mid v_{t}, \omega_{t-1}\right]-E\left[Y_{t+n} \mid \omega_{t-1}\right] \text { for } n=0,1, \ldots
$$

where $v_{t}$ is current shock and $\omega_{t-1}$ is the history.

Examples of the generalized impulse responses are illustrated in Figure 5. For this illustration, I selected a sample of 300 consecutive periods from the simulated data and estimated the $\operatorname{VAR}(4)$ and $\operatorname{RBF}(1)$. To draw impulse responses, I pick two random histories, $\omega_{t-1}$, from the sample and ensure that one of the selected histories satisfies the criterion for the normal state and the other satisfies the criterion for the zlb state. I then calculate the impulse responses with the shock occurring at these two histories.

In Figure 5, the blue solid line shows the true impulse response under the normal state, and the red solid line shows the true impulse response under the zlb state. Together with the true impulse responses, the left panels show the estimated responses from the $\operatorname{RBF}(1)$ estimator under the normal state in the blue dotted line and under the zlb state in the red dotted line. Shaded bands represent the 2.5th and 97.5th percentiles. ${ }^{13}$ The right panels show the estimated impulse responses from the linear VAR(4) with 95 percent confidence bands in black lines.

The top panels of Figure 5 show the responses of inflation rate following a positive productivity shock. The positive productivity shock is deflationary. The figure shows that the initial impact of the shock is slightly larger in magnitude during the normal state, reducing the inflation rate by $0.7 \%$ on impact. The inflation rate under the normal state then quickly returns to zero after three periods. The decline of the inflation rate is smaller under the zlb. The inflation rate goes down by $0.42 \%$ on

\footnotetext{
${ }^{12}$ Some earlier work includes Beaudry and Koop (1993), Potter (1995), Pesaran and Shin (1996), and Potter (2000). In the generalized impulse response framework, the problem of future shockdependence is handled by averaging out the impulse responses with many different future shocks. I compute the conditional expectation by the Monte Carlo integration, and the expectation is calculated by averaging out 1,000 different paths of simulated future shocks. See Koop et al. (1996) for more detail.

${ }^{13}$ The confidence bands are estimated by the residual-based block bootstrap method. When I conduct the bootstrap, the centroids and scaling parameters are re-estimated by repeating the K-means cluster method for 50 times with different initial values and by selecting the centroids that minimize the errors. My baseline preference is block bootstrap method rather than the wild bootstrap because the residuals exhibit auto-correlation. The results are almost identical when the wild bootstrap is employed.
} 
impact, and the response is slightly more persistent under the zlb. The $\mathrm{RBF}(1)$ estimator appears to be generally successful at capturing the different responses of the inflation rate under the two different states. In addition, the confidence bands are reasonably narrow even compared with the linear VAR(4) model. As expected, the linear $\operatorname{VAR}(4)$ only captures the response during the normal state.

The next panels in Figure 5 show the responses of interest rate. When the positive productivity shock creates a deflationary pressure in the economy, the central bank lowers the nominal interest rate during the normal state following the Taylor rule. The response of the interest rate is smaller during the zlb periods because of the central bank's inability to push down the interest rate any further. ${ }^{14}$ The left panel shows that the $\operatorname{RBF}(1)$ estimator is, again, successful at capturing the different interest rates responses under the two different regimes. Furthermore, the $\mathrm{RBF}(1)$ estimator is able to distinguish the two different interest rate responses under the zlb state and normal state in a statistically significant manner.

Finally, the last panels show the responses of consumption. Due to the positive productivity shock, the consumption goes up in both normal and zlb states. The consumption responses peak at the third quarter, reaching at $1.2 \%$ in the normal state and $1 \%$ in the zlb state. The weak response during the zlb periods stems from the higher real interest rate, which is caused by the absence of central bank's reaction to the deflationary pressure. As the real interest rate goes up during the zlb periods, the consumption is discouraged. The $\mathrm{RBF}(1)$ estimator seems to perform reasonably well at capturing the different consumption dynamics following the positive supply shock. In addition, the responses under the two regimes can be distinguished in a statistically significant manner.

The examples above demonstrate the potential usefulness of the RBF estimator in uncovering the nonlinear structure of the NK economy. However, since the generalized impulse responses are history-dependent, the performance of the RBF estimator could be history-dependent as well. In order to assess the overall performance and accuracy of the RBF estimator, I present below the within-sample MSEs with various histories. The MSEs are computed based on 1,000 Monte Carlo simulations. For each Monte Carlo simulation, I take the following procedures: (1) randomly pick a new sample of

\footnotetext{
${ }^{14}$ The nominal interest rates still appears to go down in the figure, but the responses shown here are relative to the counterfactual where the shock did not occur. Thus, the negative interest rates response during the zlb state is indicative that the interest rates would have increased from zero faster if the shock did not arrive.
} 
300 observations, (2) estimate the RBF and linear VARs, (3) select a history within that sample, (4) give a shock, and (5) compute the MSEs of the impulse responses. In Table 1, I present the sum of the MSEs over horizons from 1 to 30 for the normal state and zlb state, which are classified based on the level of interest rate when the shock hits the economy. In addition, I also present the MSEs for the general case where I simply calculate the MSEs from the randomly picked histories, paying no attention to whether or not the history belongs to the normal or zlb state. Top row in Table 1 shows the MSEs for the normal state, the middle row shows the MSEs for the zlb state, and the bottom row presents the MSEs for the general case.

Table 1: Average MSE Performance (estimation periods = 300)

\begin{tabular}{ccc|cc|cc}
\hline & \multicolumn{2}{c}{ Inflation } & \multicolumn{2}{c}{ Consumption } & \multicolumn{2}{c}{ Interest rates } \\
& $\operatorname{VAR}(4)$ & $\mathrm{RBF}(1)$ & $\operatorname{VAR}(4)$ & $\mathrm{RBF}(1)$ & $\operatorname{VAR}(4)$ & $\mathrm{RBF}(1)$ \\
\hline Normal state & 18.5690 & 15.2598 & 30.0534 & 17.5437 & 6.1412 & 3.5549 \\
ZLB state & 15.5559 & 10.4868 & 37.0743 & 20.9026 & 10.2899 & 4.4261 \\
General case & 18.4277 & 15.4047 & 30.8573 & 18.6588 & 6.7014 & 3.9047 \\
\hline
\end{tabular}

Notes: Summary statistics over 1000 Monte-Carlo replications. MSE is the meansquared error of the estimated impulse response function over horizons 1 to 30 .

Surprisingly, the top row of Table 1 indicates that the MSEs are smaller for the $\mathrm{RBF}(1)$ estimator for all the variables even under the normal state. This result is striking given that the NK model can be well approximated by linear functions in the normal state, and it confirms the prediction from the earlier impulse response illustrations that the RBF estimator can identify nonlinear- and history-dependent dynamic responses of the macroeconomic variables from a small sample of simulated data. Furthermore, the difference in the MSEs between the $\operatorname{RBF}(1)$ and $\operatorname{VAR}(4)$ becomes even larger for the zlb case, with errors from the $\mathrm{RBF}(1)$ as small as twothird of the MSEs of the $\operatorname{VAR}(4)$ at maximum. The result in this section indicates that the RBF estimator can be a useful alternative to the traditional linear VAR model when the form of underlying data generating process is unknown to econometricians or when it is possibly nonlinear. The result also suggests that the use of the RBF estimator for macroeconomic time series analysis may be appropriate. 


\section{Application to the US Data}

Finally, I apply the RBF estimator to the quarterly US data. Following Wieland (2016) and Garín et al. (2016), I ask if a positive supply shock is less expansionary when the nominal interest rates is at the zlb. The question is motivated by the textbook NK model that predicts a possibility of contractionary supply shock at the zlb. This somewhat counter-intuitive prediction is the extreme version of the responses studied in the previous section. I assume that a positive supply shock to be an increase in neutral productivity. An increase in productivity decreases inflation rate and the equilibrium real interest rate. When such a shock hits the economy, the central bank would react by lowering nominal interest rates according to the Taylor rule in the normal state. However, if the economy is at the zlb, the central bank is unable to push down the nominal interest rate any further. Consequently, the real interest rate will go up and the demand will suffer. In the extreme case where the zlb is expected to last for a long period, the increase in the real interest rate can be potentially so large that the negative demand response dominates the positive effect of the productivity shock. When that happens, the GDP decreases.

In contrast to the predictions of the NK model, Wieland (2016) found that the effects of a supply shock remain the same at the zlb by examining the experiences of the Great East Japan Earthquakes and the oil-supply shocks. ${ }^{15}$ Garín et al. (2016) used the utilization-adjusted TFP shock estimated by Fernald (2014) and found that a positive supply shock is even more expansionary at the zlb. As their findings potentially cast doubts on the assumptions that underlie the DSGE models, I also investigate this important question by employing the RBF estimator with the utilization-adjusted TFP shock estimated by Fernald (2014). Unlike the previous studies, I found some evidence of weaker expansionary effects during the passive monetary policy regimes.

\subsection{Data}

The dataset includes the following six variables: the log difference of (1) real GDP, $\Delta \ln Y_{t}$, (2) personal consumption, $\Delta \ln C_{t}$, (3) private investment, $\Delta \ln I_{t}$, as well as (4) $\log$ of hours worked, $\ln N_{t}$ (5) expected inflation rate from the University of

\footnotetext{
${ }^{15}$ Wieland (2016) also suggested that the expansionary effects of a supply shock may be even larger at the zlb.
} 
Michigan's Survey of Consumers, $\pi_{t}^{e}$, and (6) the effective federal funds rate, $r_{t} \cdot{ }^{16} \mathrm{In}$ addition to these variables, I use the utilization-adjusted TFP process estimated by Fernald (2014).

Garín et al. (2016) illustrated that the utilization-adjusted TFP series by Fernald (2014) can be considered an exogenous shock process. Garín et al. (2016) used four popular measures of exogenous macroeconomic shocks ${ }^{17}$ and showed that these four measures do not Granger cause the utilization-adjusted TFP shock. They, therefore, argued that the evidence is suggestive that the utilization-adjusted TFP process be treated as an exogenous shock process. Based on their evidence, I also treat the utilization-adjusted TFP process as an exogenous shock process.

The dataset begins in 1978Q1, when the quarterly data of inflation expectation became available, and ends in 2016Q4. The US economy was in the "zlb state" from the last quarter of 2008 to the last quarter of 2015.

\subsection{Generalized Impulse Responses}

I estimate the generalized impulse responses to a positive TFP shock. The estimated model is as follows:

$$
X_{t}=R B F\left(\Delta \ln A_{t},\left\{\Delta \ln A_{t-p}, X_{t-p}\right\}_{p=1}^{P}\right)+u_{t}
$$

where $X_{t}=\left[\Delta \ln Y_{t}, \Delta \ln C_{t}, \Delta \ln I_{t}, \ln N_{t}, \pi_{t}^{e}, r_{t}\right]$. I assume that the utilization-adjusted TFP shock can affect the other variables within the same period, but not vice versa. I set the lags to be four.

As in Section 4.1, I define generalized impulse response of a variable, $Y_{t+n}$, in $n$-period ahead to be:

$$
G I\left(n, v_{t}, \omega_{t-1}\right)=E\left[Y_{t+n} \mid v_{t}, \omega_{t-1}\right]-E\left[Y_{t+n} \mid \omega_{t-1}\right] \text { for } n=0,1, \ldots
$$

\footnotetext{
${ }^{16}$ Real GDP (GDPC09), personal consumption (PCEC), private investment (FPI) are from the NIPA table. Hours are obtained by multiplying civilian employment (CE16OV) and average weekly hours duration (PRS85006023). The GDP, consuption, investment, and hours are expressed as per capita by dividing the variables by civilian noninstitutional population (CNP16OV).

${ }^{17}$ The four measures are Romer and Romer (2004)'s monetary policy shocks, Romer and Romer (2010)'s tax shocks, Ramey (2011)'s defense news shock, and Kilian (2008)'s exogenous oil price shock.
} 
where $v_{t}$ is current shock, $\omega_{t-1}$ is the history, and $t$ spans from 1978Q1 to 2016Q4. I fix $v_{t}$ to be the standard deviation of $\Delta \ln A_{t}$ for all $t$. Since $\Delta \ln A_{t}$ exhibits a strong fluctuation and because the estimated impulse responses depend on the current value of $\Delta \ln A_{t}$, the resulting impulse responses also exhibit significant fluctuations. To avoid this issue, the impulse responses shown below are averaged out over $[t-$ $2, \ldots, t+2]$ for all $t$ whenever possible.

\section{$5.3 \quad$ Results}

Figures 7-8 plot the point estimates of the responses after 1 quarter, 3 quarters and 10 quarters between 1980 and 2016. In Figures 10-16, the impulse responses are shown with confidence bands for the horizons of (1) 1st quarter, (2) 2nd quarter, (3) 5th quarter, and (4) 9th quarter. In those figures, the point estimates are drawn in blue solid lines, and the 68 percent confidence bands are expressed as red dotted lines.

In general, the impulse responses from the RBF estimator look reasonably sensible. The positive supply shocks have positive and persistent effects on output, consumption, and investment. Expected inflation declines immediately after the shock, but the effect is short-lived. The Fed funds rate also decreases after the shock in most periods, which is consistent with the Taylor rule. Hour worked goes down in the first few quarters in most periods, but it rises in the longer horizon. Overall, these responses are consistent with previous studies that investigated the effects of supply shocks on macroeconomic variables. Yet, the figures also illustrate that there are substantial time-variations in the responses of the macroeconomic variables.

Figure 7(a) show the response of the cumulative GDP. ${ }^{18}$ As summarized above, the TFP shock is expansionary in all periods but exhibits a substantial time-variation in terms of the magnitude of its effects. The supply shock's after-10-quarters-effects varies from approximately $0 \%$ to $0.7 \%$ depending on the timing of the shock. The figure also suggests that the effect of productivity shock is procyclical. The 2008 Financial Crisis had a particularly powerful impact of weakening the effects of the productivity shock, reducing its after-10-quarters-effects from $0.57 \%$ in $2007 \mathrm{Q} 2$ to $0.05 \%$ in 2009Q4.

Surprisingly, the expansionary effects of the productivity shocks are considerably

\footnotetext{
${ }^{18}$ The result shown here is not GDP difference as I calculated the cumulative sum of the estimated impulse responses.
} 
weaker during the zlb periods even after the recession from the 2008 Financial Crisis was over. These weak expansionary effects are characterized by the weak on-impact effects as well as the lack of persistence. Interestingly, the similarly weak output responses are observed between 2003 and 2004, in which the persistence of the effects is also absent. The decline of the output responses is unlikely to be explained by the procyclical effects of the supply shocks since these periods do not correspond to the recessionary periods. Rather, they correspond to the recovery phase from the 2001 recession when the fed funds rate was kept very low at around $1 \%$ and also to the periods right before the rate started to rise successively in 2005 and $2006 .{ }^{19}$

Figure $7(\mathrm{c})$ plots the estimated response of the fed funds rate. While the interest rate is estimated to go down in most periods (consistent with the Taylor rule), it is estimated to either go up or stay the same between 2003 and 2004 and after the 2008 Financial Crisis. ${ }^{20}$ Furthermore, Figure $7(\mathrm{~d})$ shows the response of real interest rate calculated from the simple Fisher equation ${ }^{21}$, which indicates that the real interest rate would have been higher in response to the supply shock around 2003-2004 and after 2008. The results suggest that the response of real interest rate plays a crucial role in forming the responses of output. The fact that the supply shocks are found to have weaker expansionary effects not only during the zlb periods but also between 2003 and 2004 makes the prediction of the NK model, in which the monetary policy impotency reduce the effects of supply shocks, more convincing.

Figure 7(e) shows the response of cumulative consumption. Similar to the output response, the consumption also tend to increase in response to the positive productivity shock throughout the sample. However, the response is particularly weak during 2003-2004 periods and after the 2008 Financial Crisis. Moreover, the cumulative investment response shown in Figure $7(\mathrm{~g})$ also exhibits particularly weak responses during 2003-2004 and after 2008 periods. To reiterate, these findings are in accordance with the textbook NK model and the findings from the output and interest rates responses above. The decline of inflation expectation, combined with the unresponsive monetary policy, raises the real interest rate and discourages consumption and investment.

\footnotetext{
${ }^{19}$ see Figure 9.

${ }^{20}$ The responses during these periods are not statistically different from zero.

${ }^{21}$ The real interest rate $=$ nominal interest rate - expected inflation. Negative real interest rate response means that the fed funds rate decreased for more than one-for-one in response the the decline in the expected inflation.
} 
Finally, Figure 7(f) plots the response of the hours worked. The previous literature found that the hours worked decreases in the short-run in response to a positive productivity shock, which is often referred to as the evidence for price-stickiness in the macro models. My finding is consistent with the existing literature: the hours worked decreases in the short-run, but it rises in the longer horizon. This pattern is stable for majority of the sample periods, with the exception of the recessionary periods in the early 1980s, 2001-2005 periods, and after 2008. These periods correspond to the periods of a large slack in the labor market as well as the periods of relatively small or positive real interest rate responses. Peculiarly, the positive productivity shocks are estimated to increase the hours worked both in the short horizon and in the long horizon after 2008.

In summary, the estimated impulse responses from the RBF estimator suggest that the response of macroeconomic variables to a positive supply shock is substantially time varying. It also hints at the fact that the effects of productivity shocks might be quantitatively and qualitatively different after the recent Financial Crisis as well as 2003-2004 periods when the monetary policy is possibly less sensitive to the deflationary pressure caused by the supply shocks. In particular, the effects of the supply shocks during the periods of monetary policy inaction are found to be considerably less expansionary, which is consistent with the textbook NK model but is inconsistent with what Wieland (2016) and Garín et al. (2016) reported. The findings indicate that the economic responses to the supply shocks around 2003-2004 is markedly similar to the responses during the zlb periods and highlight the critical role that the response of real interest rate plays in determining the responses of other macroeconomic variables. Importantly, all of the findings above are based on the RBF estimator that was estimated without imposing a priori- functional form assumption.

\section{Discussion}

Why is the estimates in Section 5 starkly different from the existing literature that concludes that the supply shocks are even more expansionary under the zlb? Is the difference an artefact of the untraditional methodology of the current paper? To answer these questions, I briefly investigate the relationship between the TFP shocks and the output using the state-dependent local projection method of Jordà (2005).

Using the same data that I used in Section 5, I estimated the following regression: 


$$
\begin{aligned}
\sum_{j=0}^{h} \Delta \ln \left(Y_{t+j}\right)= & \left(1-Z_{t}\right)\left(\alpha_{h}^{n}+\beta_{h}^{n} \Delta \ln A_{t}+\phi_{h}^{n}(L) x_{t-1}\right) \\
& +Z_{t}\left(\alpha_{h}^{z}+\beta_{h}^{z} \Delta \ln A_{t}+\phi_{h}^{z}(L) x_{t-1}\right)+u_{t+h}
\end{aligned}
$$

where $Z_{t}$ is the indicator variable for the zlb periods and $x_{t}$ is the control variables. Following Garín et al. (2016), I define the zlb as the periods between 2008Q4 and 2015Q4. The control variables are lagged $\Delta \ln Y_{t}$ and $\Delta \ln A_{t}$. The number of lags are four. Since I do not need to use the expected inflation, I now extend the dataset to periods between 1948Q1 and 2016Q4. Additionally, I excluded the 2008Q4 because it nontrivially affects the estimated on-impact response of output during the zlb periods, which is likely due to the large impact of the Lehman failure and not driven by the movement in the TFP.

Figure 17 shows the estimated output responses during the normal periods in the blue line and during the zlb periods in the red line. The shaded bands represent the 90 percent confidence interval. The output response during the normal periods is initially small but grows larger over time, culminating at around $0.2 \%$ in the 10 th quarter. In contrast, the estimated response is weaker during the zlb periods and is not statistically different from zero for all horizons. The result is consistent with the finding from Section 5 that found the weaker output response when the monetary policy is not reacting actively to the shocks. As a robustness check, I also regressed the equation 10 with the dependent variable specified as $\left(\ln Y_{t+h}-\ln Y_{t-1}\right)$. The result is shown in Figure 18, and the main conclusion stays the same.

In contrast to the previous literature that concluded that the expansionary effects of the supply shocks are even larger at the zlb, I found that the supply shocks become less expansionary during the periods of zlb. The difference is likely to be a product of different data specifications rather than the methodologies. In particular, while Garín et al. (2016) used aggregate GDP and log-level specification, I used the GDP per capita and the growth rate specification. In addition, I also excluded the outlier (2008Q4). Once I treated the data as described, the supply shocks are found to have weaker expansionary effects during the passive monetary regimes even when I use the state-dependent local projection method. 


\section{Conclusion}

In this paper, I investigated the applicability of the Radial Basis Functions to the macroeconomic time series analysis. The RBF estimator can be a useful alternative to traditional estimators because of its low computational costs that allow researchers to flexibly estimate the time-varying response functions using relatively large dimensional models.

Based on a medium-scale nonlinear New Keynesian model, I performed a series of Monte Carlo experiments to study the small sample properties of the proposed RBF estimator. I found that the RBF estimator outperforms the linear VARs in terms of the within-sample MSEs. The generalized impulse responses from the RBF estimator suggests that it can learn the structure of the nonlinear New Keynesian model from the sample of simulate data whose length is as small as 300 periods.

Finally, I applied the RBF estimator to the quarterly US data and found that the responses of macroeconomic variables to a positive supply shock exhibit substantial time variations. The result also suggests that the expansionary effects of a supply shock became significantly weaker during the periods of monetary policy inaction, which is consistent with the prediction of a textbook NK model.

The results shown in this paper highlights the potential benefit and validity of the RBF estimator in analyzing the macroeconomic time series data. The estimator is especially useful when econometricians do not have a clear understanding of the underlying data-generating process. 


\section{Appendix}

\subsection{NK Model for Monte Carlo simulation}

The economy is inhabited by four types of agents: Households, Final good producer, Intermediate goods producers, and Monetary authority. Except for the habit persistence in the household sector, the following NK economy is standard.

\subsubsection{Households with Habit Persistence}

There is a continuum of households who consume a composite good, $C_{t}$, supply labor, $N_{t}$, and purchase bond $B_{t}$. The representative household maximizes the expected lifetime utility given by:

$$
E_{t}\left[\sum_{t=1}^{\infty} \beta^{t-1}\left\{\frac{\left(C_{t}-\gamma C_{t-1}\right)^{1-\sigma}}{1-\sigma}+\frac{\left(1-N_{t}\right)^{1-\kappa}}{1-\kappa}\right\}\right]
$$

where $\gamma$ controls the degree of habit persistence, and $\beta$ is the discount factor.

The household is subject to the following budget constraint each period:

$$
P_{t} C_{t}+\frac{1}{R_{t}} B_{t}=W_{t} N_{t}+B_{t-1}+P_{t} \Pi_{t}
$$

where $P_{t}, W_{t}$, and $R_{t}$ are commodity good price, nominal wage, and nominal interest rates, respectively. In addition, $\Pi_{t}$ is the profit from the intermediate-good firms.

We can maximize utility subject to the budget constraint to obtain the optimal allocation of consumption across time:

$$
\lambda_{t}=\beta E_{t}\left[\lambda_{t+1} R_{t} / \pi_{t+1}\right]
$$

where $\pi_{t+1}=P_{t+1} / P_{t}$ and $\lambda_{t}=\left(C_{t}-\gamma C_{t-1}\right)^{-\sigma}$.

The first order condition concerning labor supply decision is

$$
W_{t}=\left(\left(1-N_{t}\right)^{-\kappa} / \lambda_{t}\right)
$$




\subsubsection{Final Good Producer}

There are perfectly competitive final good producers who use intermediate goods, $Y_{t}(i)$, for $i \in[0,1]$ as inputs and produce final good, $Y_{t}$, at a price $P_{t}$ to maximize the profit given by:

$$
\max _{Y_{t}(i)} P_{t} Y_{t}-\int_{0}^{1} P_{t}(i) Y_{t}(i) d i
$$

The technology of the final good producer is given by the following CES aggregator:

$$
Y_{t}=\left(\int_{0}^{1} Y_{t}(i)^{\frac{(\eta-1)}{\eta}} d i\right)^{\frac{\eta}{(\eta-1)}}
$$

where $Y_{t}(i)$ and $P_{t}(i)$ are quantity and price of an intermediate good $i$, respectively.

\subsubsection{Intermediate Goods Producers}

There is a continuum of monopolistically competitive intermediate goods producers who uses labor, $N_{t}(i)$, as an input and solve the following cost minimization problem:

$$
\min _{N_{t}(i)} T C\left(Y_{t}(i)\right)=W_{t} N_{t}(i)
$$

where $T C$ is nominal total cost. The production technology of the intermediate goods producers are the following:

$$
Y_{t}(i)=A_{t} N_{t}(i)
$$

where $A_{t}$ is a productivity shock that follows the $\mathrm{AR}(1)$ process given by:

$$
\log \left(A_{t}\right)=\rho_{a} \log \left(A_{t-1}\right)+\epsilon_{t}^{a}, \quad \epsilon_{t}^{a} \backsim N\left(0, \sigma_{a}^{2}\right)
$$

The cost minimization problem of firm $i$ implies

$$
m c_{t}=\frac{W_{t}}{P_{t} A_{t}}
$$

where $m c_{t}$ is the Lagrange multiplier and also the real marginal cost of production.

The intermediate goods producers are subject to Calvo-type price setting friction. In this environment, only a $1-\xi$ fraction of the firms set prices optimally each period: $P_{t}(i)=P_{t}^{*}$, and the remaining fraction $\xi$ of the firms are not allowed to change the 
price, $P_{t}(i)=P_{t-1}(i)$. The profit maximization problem of a re-optimizing firm $i$, who takes into account the probability of adjusting its price next period and onwards, is given by the following:

$$
P_{t}^{*} \sum_{j=0}^{\infty} \beta^{j} \xi^{j} E_{t}\left\{\Lambda_{t+j}\left[P_{t}^{*} Y_{t+j}(i)-P_{t+j} m c_{t+j} Y_{t+j}(i)\right]\right\}
$$

where $\Lambda_{t}$ is the household's marginal utility of wealth at period $t$. I assume that the intermediate good producers are owned by the household, and all the profits are transferred to the households. The intermediate goods producers solve the optimization problem described above subject to the demand curve for their own goods, which is given by the following:

$$
Y_{t}(i)=Y_{t}\left(\frac{P_{t}(i)}{P_{t}}\right)^{-\eta}
$$

\subsubsection{Monetary Authority}

Lastly, a monetary authority sets nominal interest rate according to the Taylor rule as follows:

$$
R_{t}=\max \left[\frac{\bar{\pi}}{\beta}\left(\frac{R_{t-1}}{\bar{\pi} / \beta}\right)^{\phi_{R}}\left(\left(\frac{\pi_{t}}{\bar{\pi}}\right)^{\phi_{\pi}}\left(\frac{Y_{t}}{\bar{Y}}\right)^{\phi_{y}}\right)^{1-\phi_{R}}, 1\right]
$$

where $\bar{\pi}$ is inflation target, $\pi_{t}$ is inflation rate between $t-1$ and $t$, and $\bar{Y}$ is the output target. This monetary authority is subject to the zero lower bound, meaning that the monetary authority cannot set nominal interest rate below zero (or equivalently, $R \geq 1)$.

\subsubsection{Aggregate conditions}

The aggregate resource constraint is simply given by

$$
C_{t}=Y_{t}
$$

In the Calvo pricing setting, firms that change prices in different periods will have different prices. Therefore, the economy needs to track price dispersion. When firms have different relative prices, there are distortions that create a wedge between the aggregate output measured in terms of production factor inputs and aggregate demand 
measured in terms of composite goods. Specifically,

$$
A_{t} N_{t}(i)=Y_{t}(i)=\left(\frac{P_{t}(i)}{P_{t}}\right)^{-\eta} Y_{t}
$$

which implies, in aggregate,

$$
N_{t}=\int_{0}^{1} N_{t}(i) d i=\frac{Y_{t}}{A_{t}} \int_{0}^{1}\left(\frac{P_{t}(i)}{P_{t}}\right)^{-\eta} d i=\frac{Y_{t} v_{t}}{A_{t}}
$$

where price dispersion, $v_{t}$, can be described as:

$$
v_{t} \equiv \int_{0}^{1}\left(\frac{P_{t}(i)}{P_{t}}\right)^{-\eta} d i=\xi \pi_{t}^{\eta} v_{t-1}+(1-\xi)\left(\frac{P_{t}^{*}}{P_{t}}\right)^{-\eta}
$$

\subsection{Equilibrium conditions}

I summarize below the first order conditions that characterize the equilibrium of our economy. Let $p_{t}^{*}=P_{t}^{*} / P_{t}$ and $a_{t}=\log \left(A_{t}\right)$. Then:

$$
\begin{aligned}
\lambda_{t} & =\left(C_{t}-\gamma C_{t-1}\right)^{-\sigma} \\
\lambda_{t} & =\beta E_{t}\left[\lambda_{t+1} R_{t} /\left(\pi_{t+1}\right)\right] \\
v_{t} Y_{t} & =a_{t} N_{t} \\
m c_{t} & =\left(\left(1-N_{t}\right)^{-\kappa} / \lambda_{t}\right) / a_{t} \\
C_{t} & =Y_{t} \\
R_{t} & =\max \left[\frac{\bar{\pi}}{\beta}\left(\frac{R_{t-1}}{\bar{\pi} / \beta}\right)^{\phi_{R}}\left(\left(\frac{\pi_{t}}{\bar{\pi}}\right)^{\phi_{\pi}}\left(\frac{Y_{t}}{\bar{Y}}\right)^{\phi_{y}}\right)^{1-\phi_{R}}, 1\right] \\
p_{t}^{*} & \left.=\left(\left(1-\xi \pi^{\eta-1}\right) /(1-\xi)\right)^{1 /(1-\eta)}\right] \\
v_{t} & =\xi \pi_{t}^{\eta} v_{t-1}+(1-\xi) p_{t}^{*-\eta} \\
S_{t} & =\lambda_{t} m c_{t} Y_{t}+\beta \xi E_{t}\left[\pi_{t+1}^{\eta} S_{t+1}\right] \\
F_{t} & =\lambda_{t} Y_{t}+\beta \xi E_{t}\left[\pi_{t+1}^{\eta-1} F_{t+1}\right] \\
p_{t}^{*} & =S_{t} / F_{t}
\end{aligned}
$$




\subsection{Numerical solution of nonlinear NK model}

The zero lower bound in the Taylor rule introduces the nonlinearity in the NK model. Thus, the solution to the equilibrium condition must be obtained using a global solution method. I use the projection method. Let $S=\left[v_{t}, C_{t-1}, R_{t-1}, a_{t}\right]$ be the state variables of the model. There are three future variables, $\left[\pi_{t+1}(S), Y_{t+1}(S), F_{t+1}(S)\right]$, that need to be interpolated. I approximate each of future variables with the Radial Basis Functions, $\left[\pi_{t+1}(\tilde{S}), Y_{t+1}(\tilde{S}), F_{t+1}(\tilde{S})\right]$, in such a way that equilibrium conditions of the model are satisfied at a set of collocation points, $\tilde{S}$. These collocation points, $\tilde{S}$, are selected based on the Maliar and Maliar (2015)'s Epsilon-Distinguishable Set algorithm. The solution's accuracy is presented in the Appendix 8.5. The model is calibrated to a set of standard parameter values in the NK literature. The calibrated parameter values are listed in the Appendix 8.4.

The algorithm for the numerical solution of the model is given below. There are two loops in the algorithm. The outer loop iterates over the grid, and the inner loop iterates over policy functions.

Step 0a: Solve the log-linear version of the model and simulate data. This initial step is required for the clustering methods in Step 1.

Step 0b: Defining the grid and the polynomials of the RBF. Given the simulated data, construct a grid following Maliar and Maliar (2015) and estimate the $\mathrm{RBF}$ coefficients for the policy functions.

Step 1: Computing integrals. Compute the integrals using the Maliar and Maliar (2015)

Step 2: Equilibrium conditions. For each grid points, use the polynomials obtained in Step 1 to compute the values of future variables, $\left[\pi_{t+1}(\tilde{S}), Y_{t+1}(\tilde{S}), F_{t+1}(\tilde{S})\right]$. Given the future variables, solve for the endogenous state variables next period using the model's equilibrium conditions.

Step 3: Evaluate conditional expectations. Using the integrals computed in Step 1, evaluate the conditional expectations in equations $23,29,30$.

Step 4: Evaluate new policy functions. Given the conditional expectations, obtain new values of future variables in the current period, $\left[\pi_{t}^{\prime}(\tilde{S}), Y_{t}^{\prime}(\tilde{S}), F_{t}^{\prime}(\tilde{S})\right]$, using equations 23, 29, 30. Given these new values, compute the new policy functions, 
and compute the difference between the polynomials of newly obtained policy functions and those of old policy functions. Denote the percentage difference as $r$.

Step 5: Iteration. If $r<10^{-8}$, go to Step 6. Otherwise, update the guess and repeat Step 1-5.

Step 6: Compute new grid. Using the solution obtained in the previous steps, simulate new data. Using these simulated data, choose a new grid using Maliar and Maliar (2015). Compute the difference between the old grid and the new grid. Specifically, for each newly computed grid point, find the nearest point in the old grid and compute the Euclidean distance. This forms a vector, $D$, that contains the distances between each new grid point to its nearest point in the old grid. Find the maximum of $D$ and call it $r_{g}$.

Step 7: Iteration for grid. If $r_{g}$ is smaller than the Euclidean distance between the farthest two points in the old grid, stop the algorithm. Otherwise, go back to Step 2 with the new grid obtained in Step 6 . 


\subsection{Calibration}

The parameter values for calibration of the New Keynesian model are summarized as follows:

Table 2: Calibration

\begin{tabular}{ccc}
\hline parameter & value & description \\
\hline$\gamma$ & 0.5 & Habit Persistence \\
$\beta$ & 0.99 & Discount factor \\
$\sigma$ & 1 & Utility Curvature: Consumption \\
$\kappa$ & 2.65 & Utility Curvature: Leisure \\
$\xi$ & 0.60 & Calvo: $(1-\xi) \%$ adjust prices each period \\
$\eta$ & 9 & Price Elasticity of Demand \\
$\bar{\pi}$ & 1 & Inflation Target \\
$\phi_{\pi}$ & 2.21 & Taylor Rule inflation coefficient \\
$\phi_{y}$ & 0.07 & Taylor Rule output gap coefficient \\
$\phi_{R}$ & 0.82 & Interest Rate Smoothing \\
$\rho_{a}$ & 0.80 & Prod. shock persistence \\
$\sigma_{a}$ & 0.019 & Prod. shock std. \\
\hline
\end{tabular}

\subsection{Accuracy of numerical solution}

I check the accuracy of the numerical solution by computing the errors of the residual equations. Specifically, I proceed as follows. First, I simulate the model forward for 10,000 periods. This gives a simulation for both the state and control variables of the model for 10,000 periods. Second, compute the residuals from the intertemporal equations 23, 29, 30 for the 10,000 periods. I report the decimal log of the absolute value of these residual errors.

On average, residual equation errors are on order of -3.53 for equation $23,-4.94$ for equation 29 , and -2.58 for equation 30 . These numbers are comparable to the other studies whose models have similar degrees of complexity. 


\section{References}

Regis Barnichon and Christian Matthes. Gaussian mixture approximations of impulse responses and the non-linear effects of monetary shocks. 2016. 10

Paul Beaudry and Gary Koop. Do recessions permanently change output? Journal of Monetary economics, 31(2):149-163, 1993. 13

David S Broomhead and David Lowe. Radial basis functions, multi-variable functional interpolation and adaptive networks. Complex System, 2:321-355, 1988. 5

Fabio Canova. Modelling and forecasting exchange rates with a bayesian time-varying coefficient model. Journal of Economic Dynamics and Control, 17(1-2):233-261, 1993. 2

Sheng Chen, Colin FN Cowan, and Peter M Grant. Orthogonal least squares learning algorithm for radial basis function networks. IEEE Transactions on neural networks, 2(2):302-309, 1991. 7

Timothy Cogley and Thomas J Sargent. Evolving post-world war ii us inflation dynamics. NBER macroeconomics annual, 16:331-373, 2001. 2

John G Fernald. A quarterly, utilization-adjusted series on total factor productivity. Federal Reserve Bank of San Francisco, 2014. 3, 16, 17

Jerome Friedman, Trevor Hastie, and Robert Tibshirani. The elements of statistical learning. Springer series in statistics New York, second edition, 2008. 8, 9

Julio Garín, Robert Lester, and Eric Sims. Are supply shocks contractionary at the zlb? evidence from utilization-adjusted tfp data. Technical report, National Bureau of Economic Research, 2016. 4, 16, 17, 20, 21

Federico Girosi and Tomaso Poggio. Networks and the best approximation property. Biological cybernetics, 63(3):169-176, 1990. 5

Erkam Guresen, Gulgun Kayakutlu, and Tugrul U Daim. Using artificial neural network models in stock market index prediction. Expert Systems with Applications, 38(8):10389-10397, 2011. 11 
James D Hamilton. A new approach to the economic analysis of nonstationary time series and the business cycle. Econometrica: Journal of the Econometric Society, pages $357-384,1989.9$

Eric J Hartman, James D Keeler, and Jacek M Kowalski. Layered neural networks with gaussian hidden units as universal approximations. Neural computation, 2(2): 210-215, 1990. 2

Kirstin Hubrich and Timo Teräsvirta. Thresholds and smooth transitions in vector autoregressive models. In VAR Models in Macroeconomics-New Developments and Applications: Essays in Honor of Christopher A. Sims, pages 273-326. Emerald Group Publishing Limited, 2013. 9

James M Hutchinson, Andrew W Lo, and Tomaso Poggio. A nonparametric approach to pricing and hedging derivative securities via learning networks. The Journal of Finance, 49(3):851-889, 1994. 11

Òscar Jordà. Estimation and inference of impulse responses by local projections. The American Economic Review, 95(1):161-182, 2005. 10, 20

Lutz Kilian. Exogenous oil supply shocks: how big are they and how much do they matter for the us economy? The Review of Economics and Statistics, 90(2):216240, 2008. 17

Lutz Kilian and Helmut Lütkepohl. Structural vector autoregressive analysis. Cambridge University Press, 2017. 2, 10

Gary Koop, M Hashem Pesaran, and Simon M Potter. Impulse response analysis in nonlinear multivariate models. Journal of econometrics, 74(1):119-147, 1996. 13

Chung-Ming Kuan and Halbert White. Artificial neural networks: An econometric perspective. Econometric reviews, 13(1):1-91, 1994. 11

Gerson Lachtermacher and J David Fuller. Back propagation in time-series forecasting. Journal of forecasting, 14(4):381-393, 1995. 11

Lilia Maliar and Serguei Maliar. Merging simulation and projection approaches to solve high-dimensional problems with an application to a new keynesian model. Quantitative Economics, 6(1):1-47, 2015. 27, 28 
John Moody and Christian J Darken. Fast learning in networks of locally-tuned processing units. Neural computation, 1(2):281-294, 1989. 5

Saeed Moshiri and Norman Cameron. Neural network versus econometric models in forecasting inflation. 1999. 11

Jooyoung Park and Irwin W Sandberg. Universal approximation using radial-basisfunction networks. Neural computation, 3(2):246-257, 1991. 2, 5

M Hashem Pesaran and Yongcheol Shin. Cointegration and speed of convergence to equilibrium. Journal of econometrics, 71(1):117-143, 1996. 13

Tomaso Poggio and Federico Girosi. Networks for approximation and learning. Proceedings of the IEEE, 78(9):1481-1497, 1990. 5

Simon M Potter. A nonlinear approach to us gnp. Journal of applied econometrics, 10(2):109-125, 1995. 13

Simon M Potter. Nonlinear impulse response functions. Journal of Economic Dynamics and Control, 24(10):1425-1446, 2000. 13

Giorgio E Primiceri. Time varying structural vector autoregressions and monetary policy. The Review of Economic Studies, 72(3):821-852, 2005. 2, 10

Valerie A Ramey. Identifying government spending shocks: it's all in the timing. The Quarterly Journal of Economics, 126(1):1-50, 2011. 17

Christina D Romer and David H Romer. A new measure of monetary shocks: Derivation and implications. American Economic Review, pages 1055-1084, 2004. 17

Christina D Romer and David H Romer. The macroeconomic effects of tax changes: Estimates based on a new measure of fiscal shocks. American Economic Review, 100:763-801, 2010. 17

Christopher A Sims. A nine-variable probabilistic macroeconomic forecasting model. In Business Cycles, Indicators and Forecasting, pages 179-212. University of Chicago Press, 1993. 2

James H Stock and Mark W Watson. Evidence on structural instability in macroeconomic time series relations. Journal of Business \& Economic Statistics, 14(1): 11-30, 1996. 2 
Norman R Swanson and Halbert White. A model selection approach to real-time macroeconomic forecasting using linear models and artificial neural networks. Review of Economics and Statistics, 79(4):540-550, 1997. 11

Greg Tkacz. Neural network forecasting of canadian gdp growth. International Journal of Forecasting, 17(1):57-69, 2001. 11

Johannes Wieland. Are negative supply shocks expansionary at the zero lower bound? 2016. $4,16,20$

Lei Xu, Adam Krzyżak, and Alan Yuille. On radial basis function nets and kernel regression: statistical consistency, convergence rates, and receptive field size. Neural Networks, 7(4):609-628, 1994. 5

Gioqinang Zhang and Michael Y Hu. Neural network forecasting of the british pound/us dollar exchange rate. Omega, 26(4):495-506, 1998. 11

Yan-Qing Zhang and Xuhui Wan. Statistical fuzzy interval neural networks for currency exchange rate time series prediction. Applied Soft Computing, 7(4):11491156, 2007. 11 
Figure 5: Examples of Generalized Impulse Responses

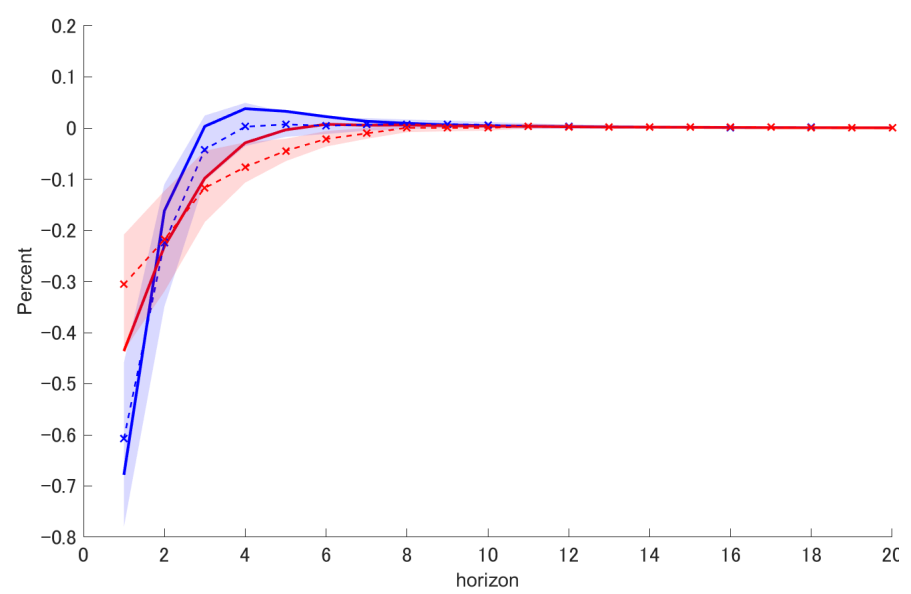

Inflation: $\mathrm{RBF}(1)$

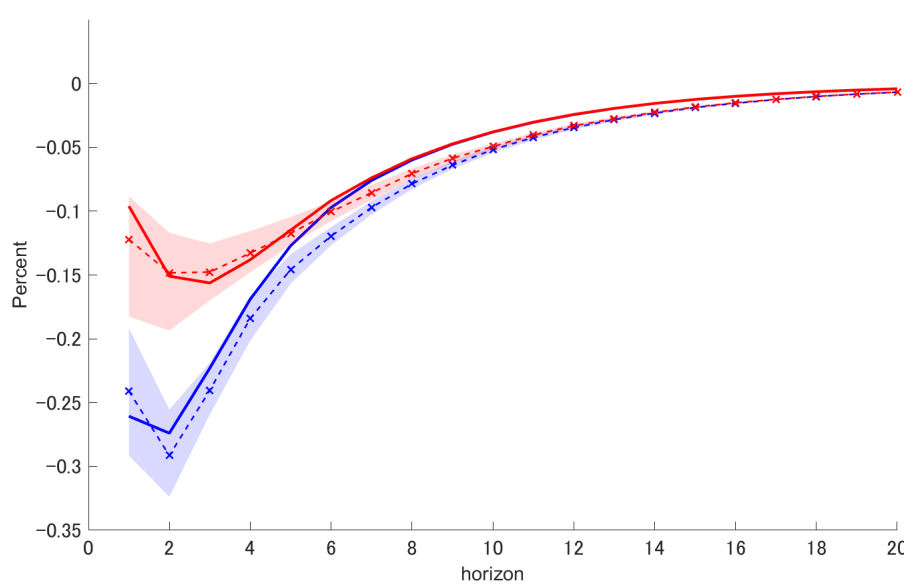

Interest Rate: $\mathrm{RBF}(1)$

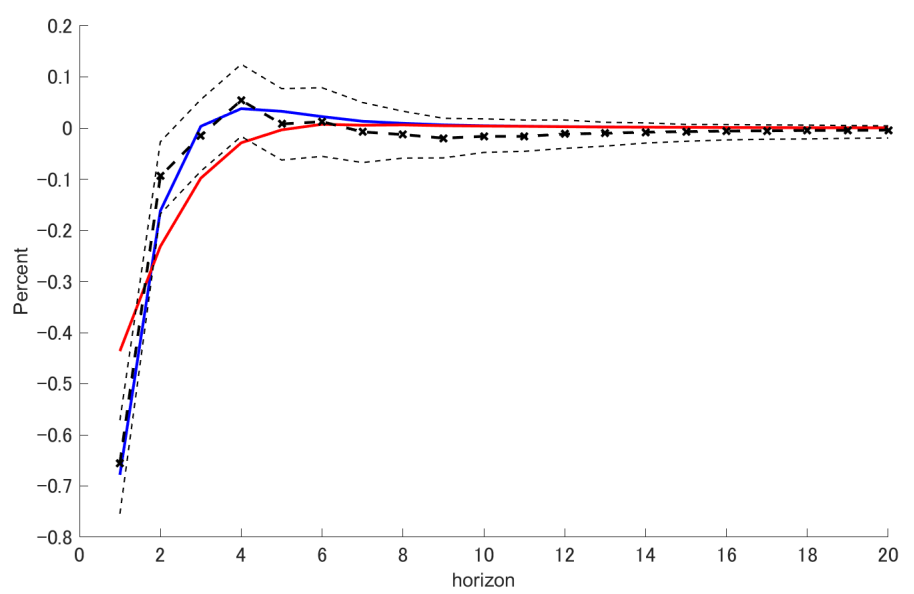

Inflation: $\operatorname{VAR}(4)$

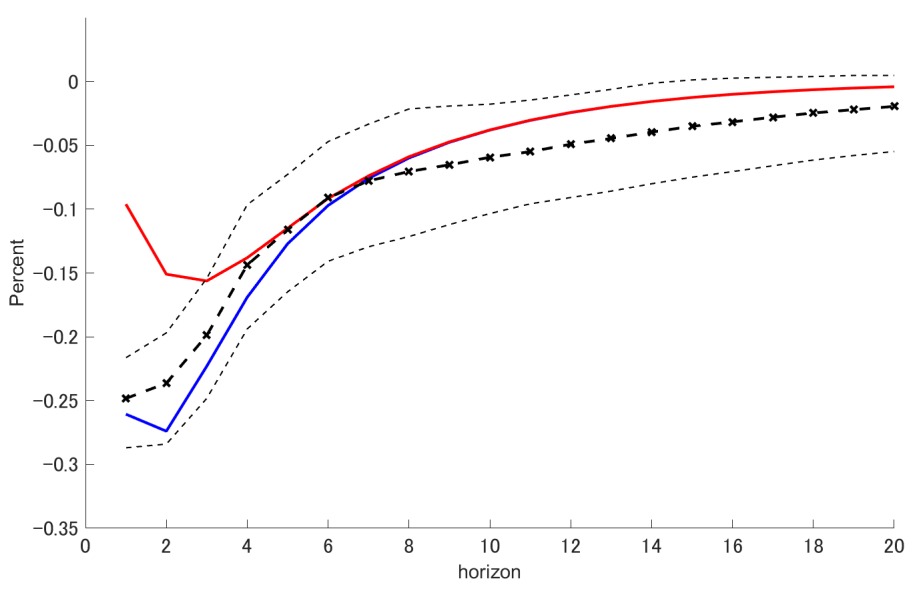

Interest Rate: $\operatorname{VAR}(4)$ 
Figure 6: Examples of Generalized Impulse Responses

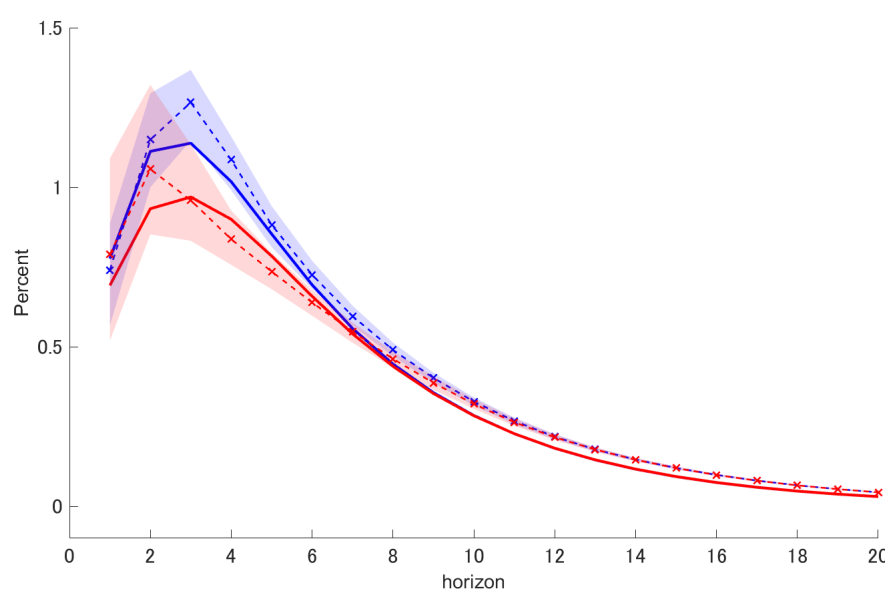

Consumption: $\operatorname{RBF}(1)$

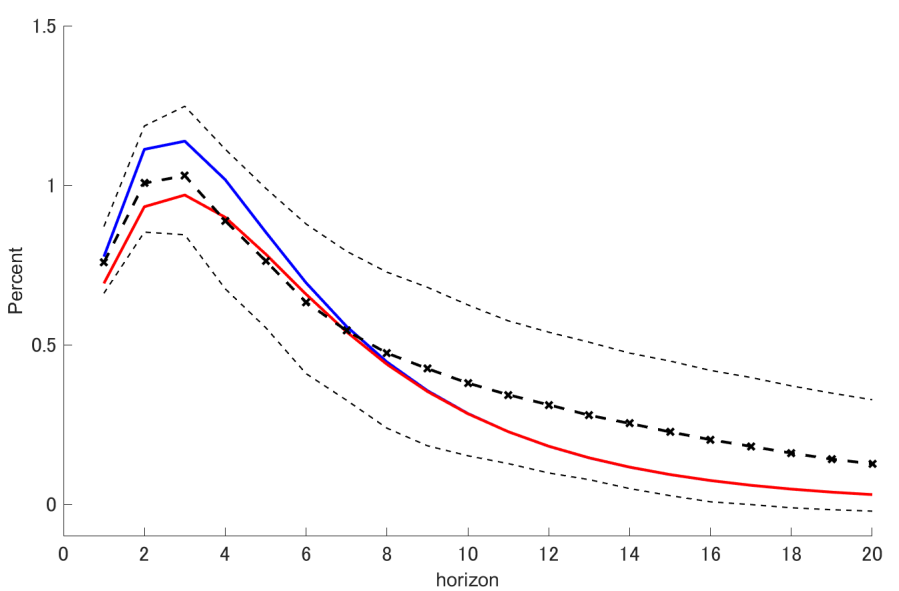

Consumption: $\operatorname{VAR}(4)$

Notes: Impulse response functions of the inflation rate, interest rate, and consumption (in percent) to a one standard-deviation positive productivity shock. True structural impulse responses from the NK model in normal state (blue solid line) or zlb state (red solid line). Estimation from a RBF(1) in normal state (blue dashed line) or zlb state (red dashed line). Estimated impulse response from a VAR(4) (black dashed line). Shaded bands denote the 2.5th and 97.5th percentiles estimated by the residual-based block bootstrap method. 
Figure 7: Responses at different horizons

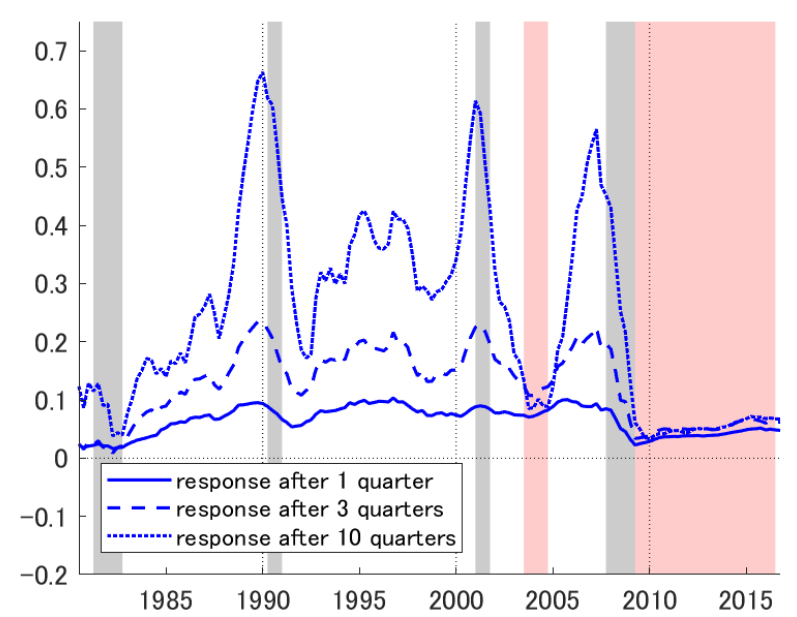

(a) cumulative GDP

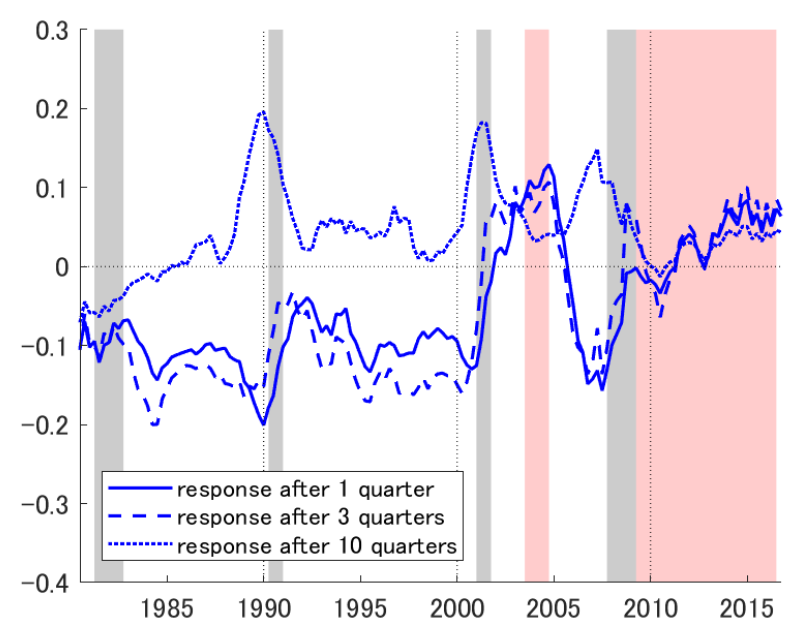

(c) Fed funds rate

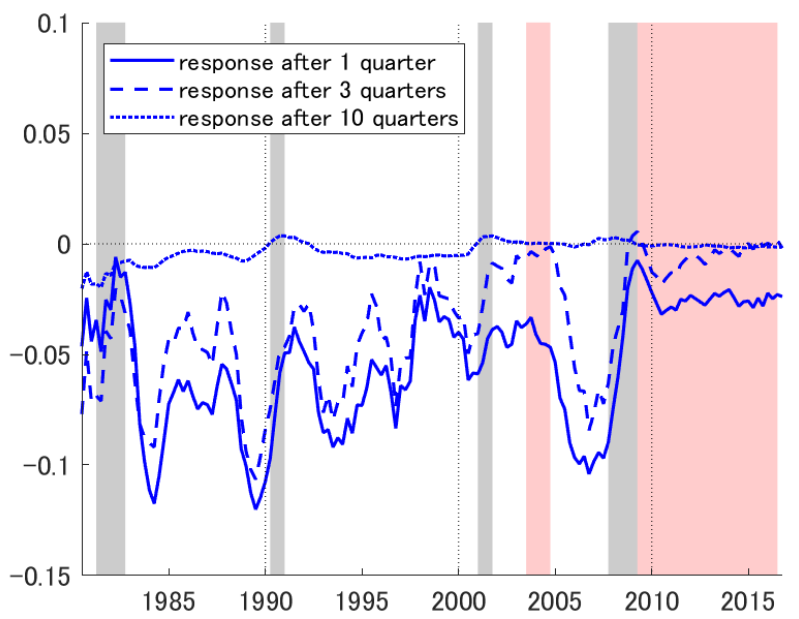

(b) Expected Inflation

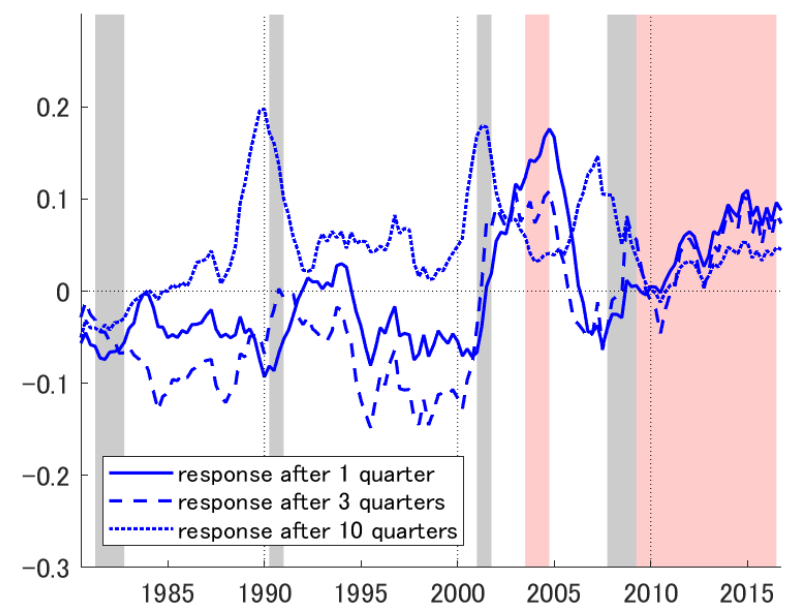

(d) real interest rate

Notes: Impulse response functions (in percent) to a one standard-deviation positive TFP shock after 1st, 3rd, and 10th quarters. Black shaded areas corresponds to NBER-recession dates. Red shaded areas represent the periods of weak output responses outside the recession dates. 
Figure 8: Responses at different horizons

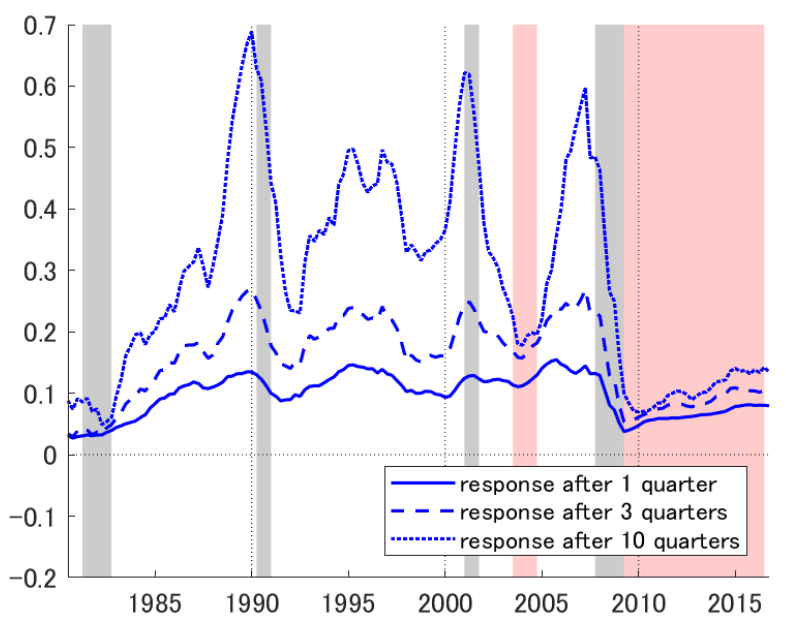

(e) cumulative Consumption

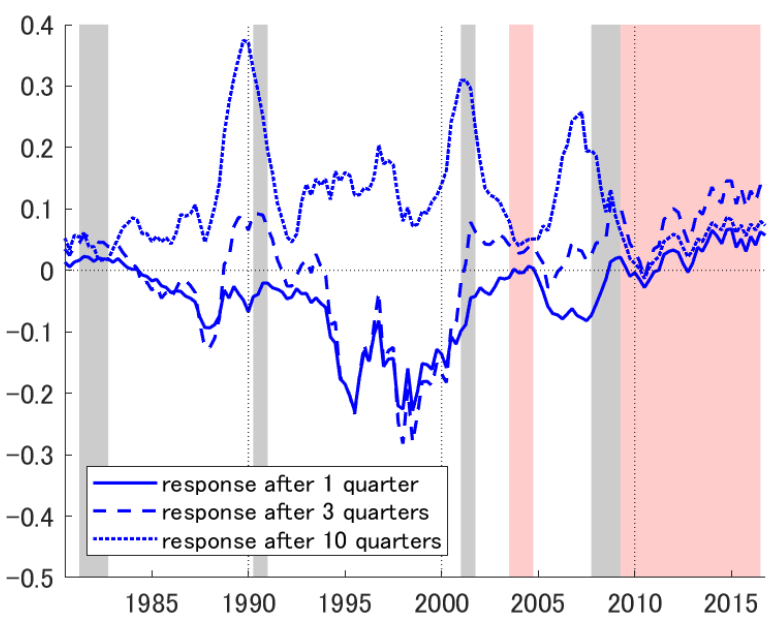

(f) Hours worked

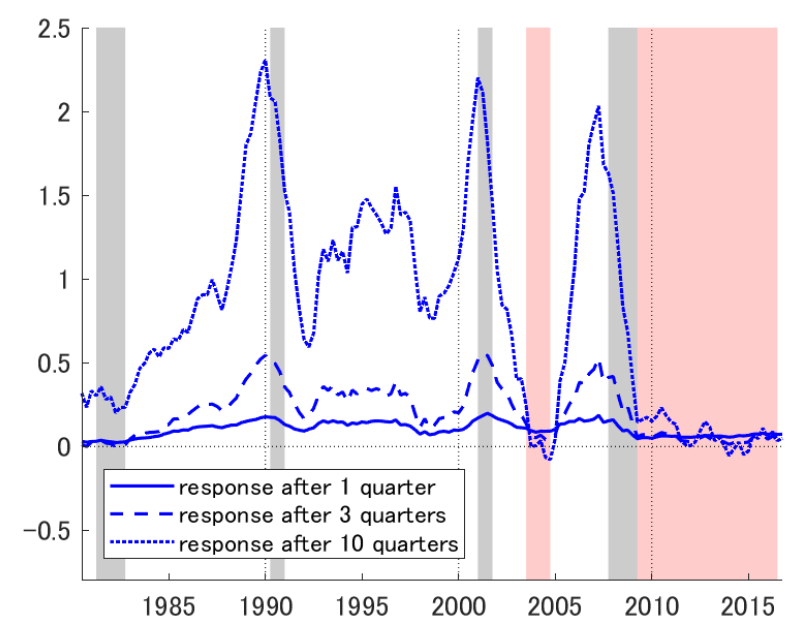

(g) cumulative Investment

Notes: Impulse response functions (in percent) to a one standard-deviation positive TFP shock after 1st, 3rd, and 10th quarters. Black shaded areas corresponds to NBER-recession dates. Red shaded areas represent the periods of weak output responses outside the recession dates. 
Figure 9: Federal funds rate

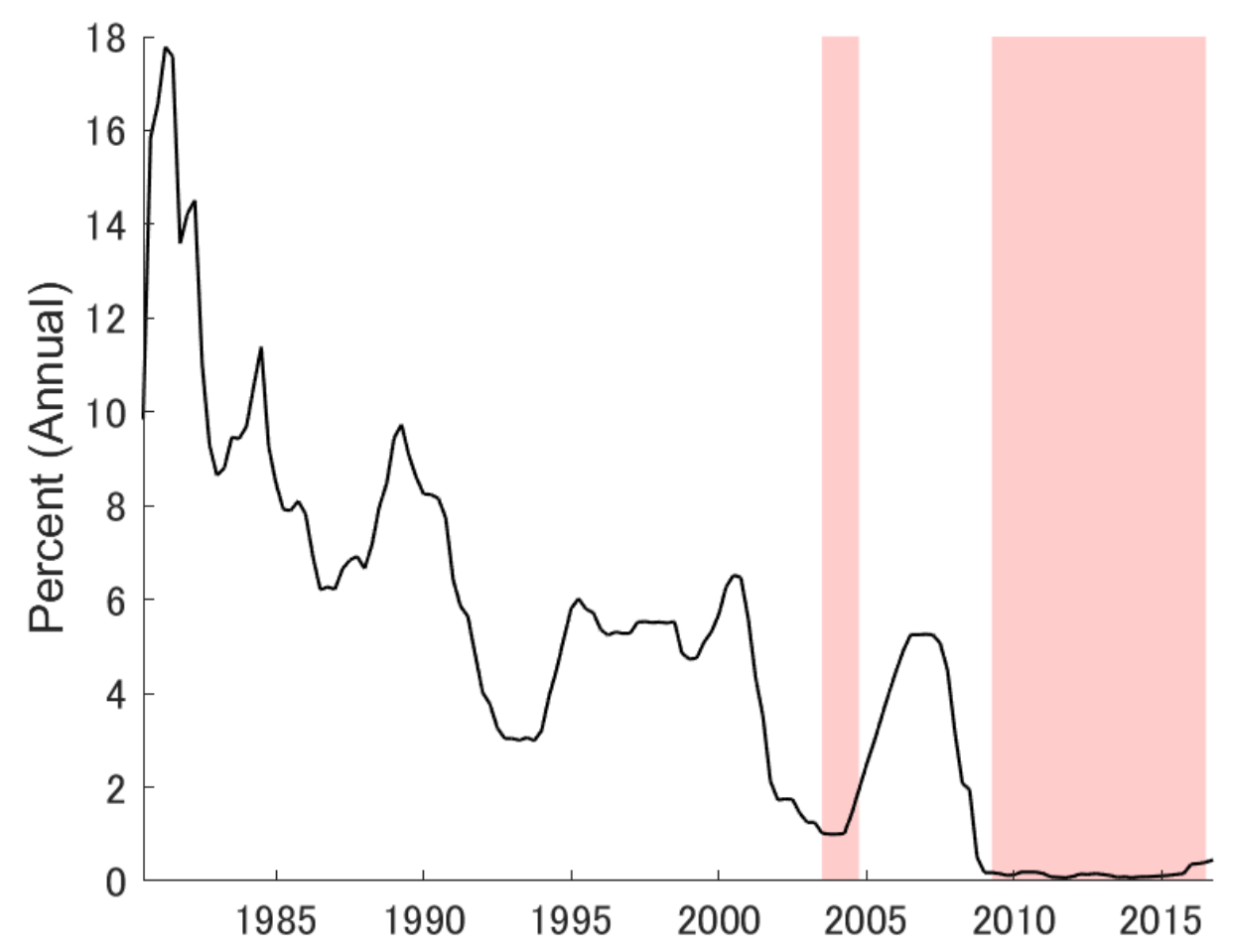

Notes: Federal funds rate between 1980 and 2016. Red shaded areas represent the periods of weak output responses outside the recession dates. 
Figure 10: Responses of cumulative GDP

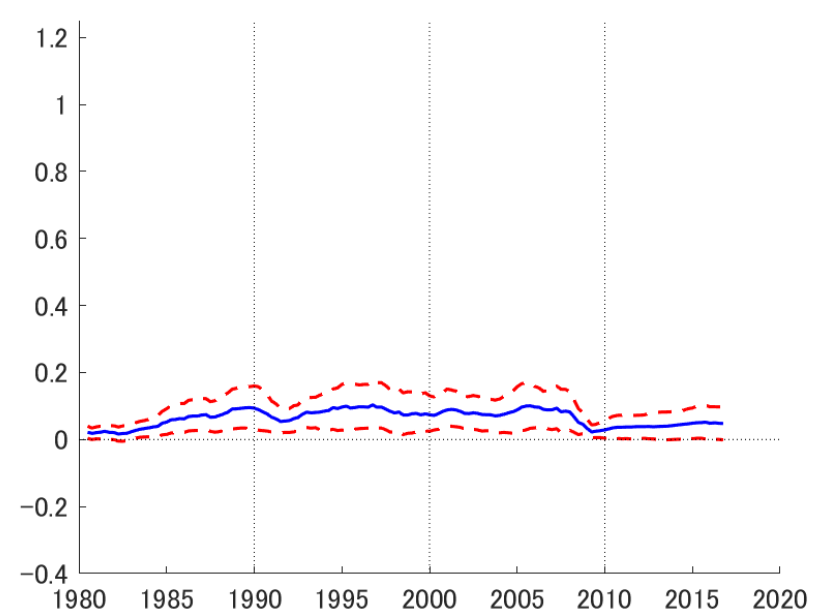

(a) Response after 1st quarter

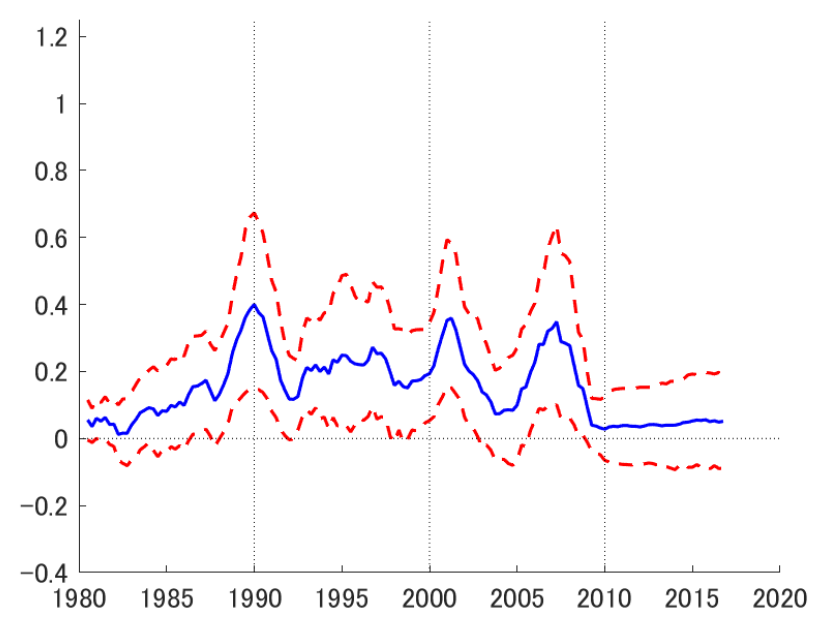

(c) Response after 5th quarter

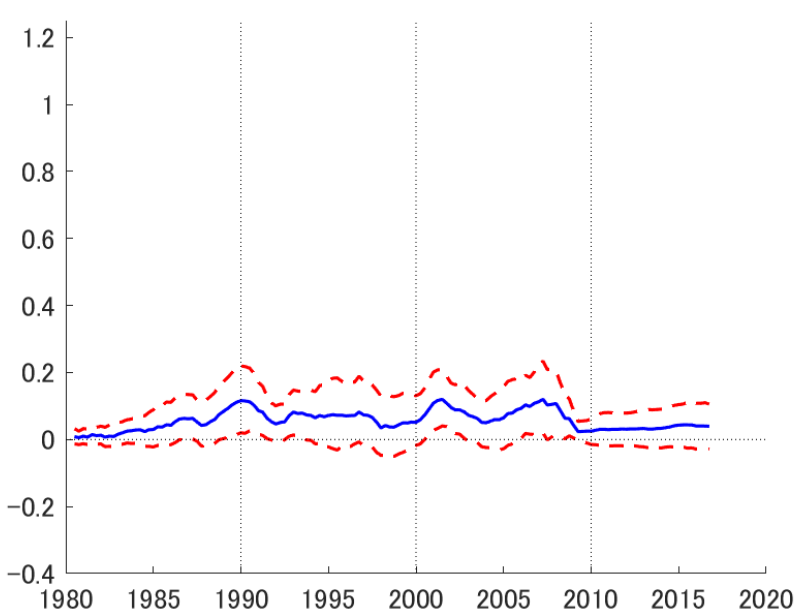

(b) Response after 2nd quarter

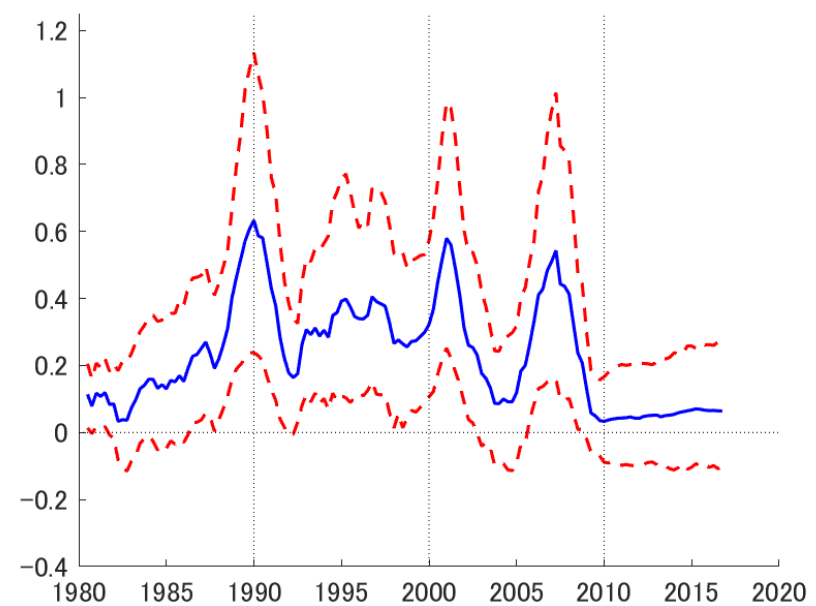

(d) Response after 9th quarter

Notes: Impulse response functions of the cumulative real GDP (in percent) to a one standarddeviation positive TFP shock. Shaded bands denote the $68 \%$ confidence interval estimated by the residual-based block bootstrap method. 
Figure 11: Responses of Expected Inflation

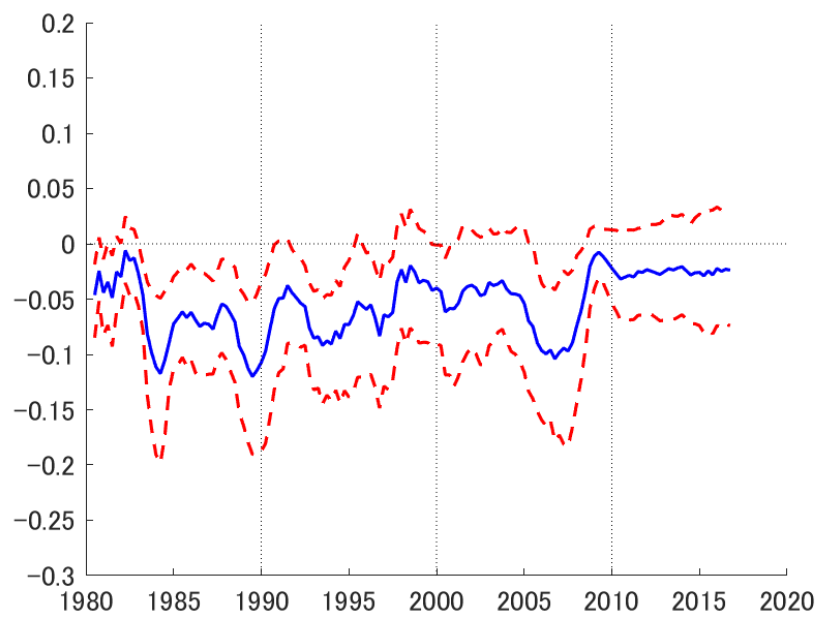

(a) Response after 1st quarter

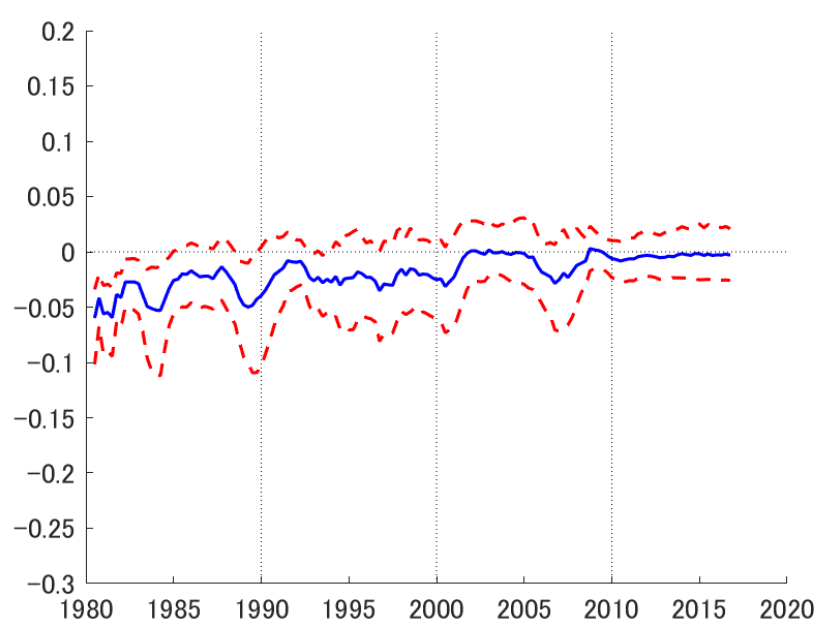

(c) Response after 5th quarter

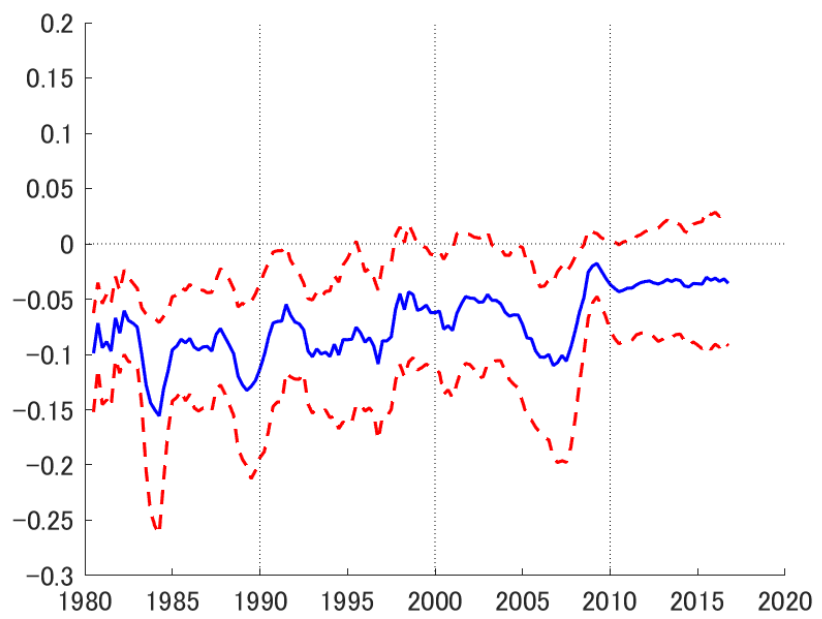

(b) Response after 2nd quarter

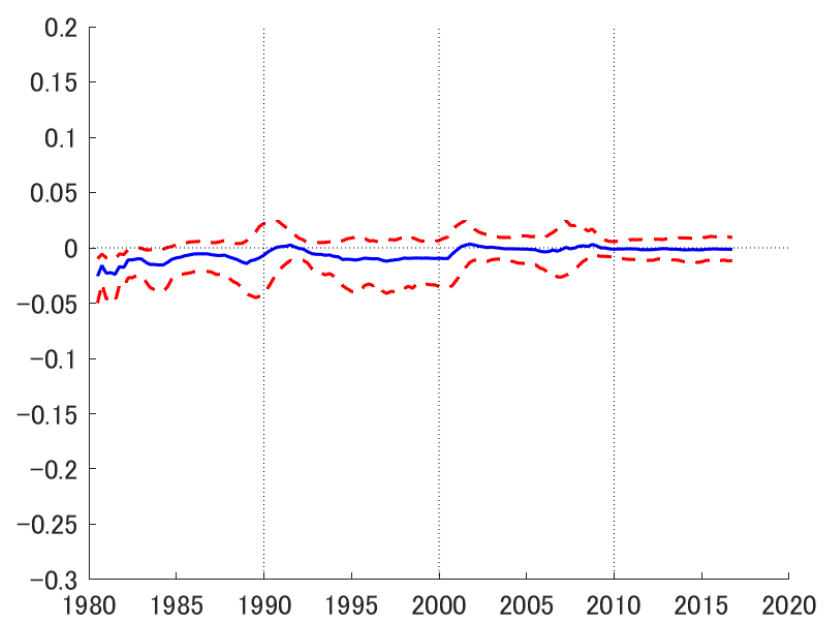

(d) Response after 9th quarter

Notes: Impulse response functions of the expected inflation (in percent) to a one standard-deviation positive TFP shock. Dotted lines show the $68 \%$ confidence bands estimated by the residual-based block bootstrap method. 
Figure 12: Responses of Fed Funds rate

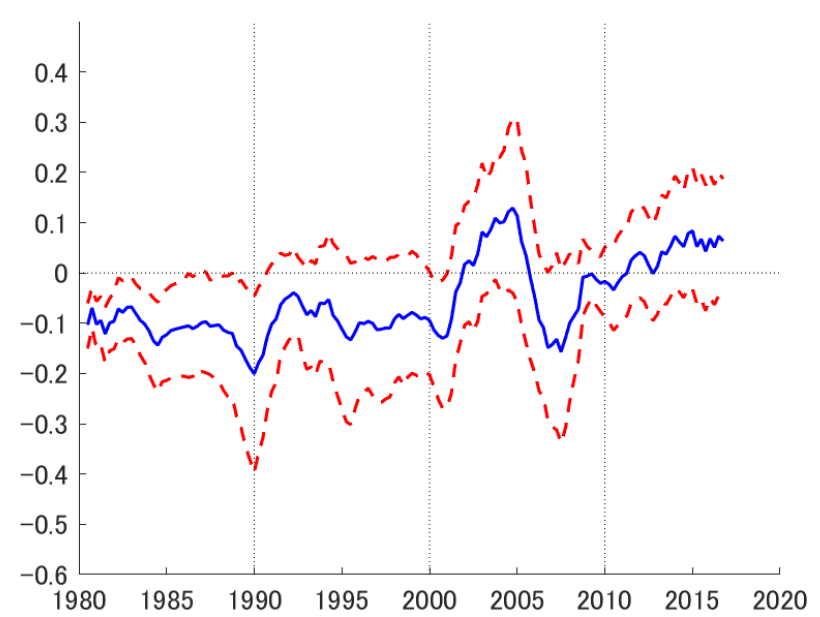

(a) Response after 1st quarter

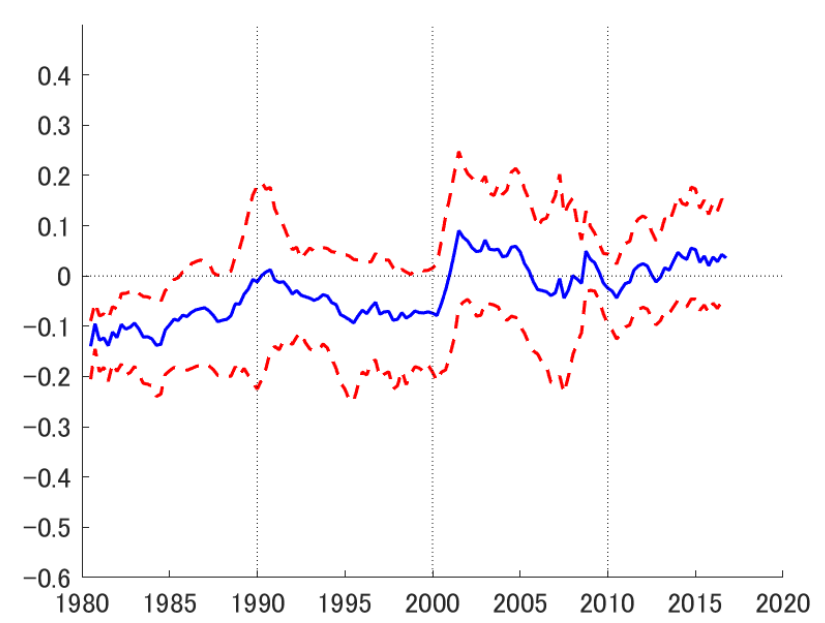

(c) Response after 5th quarter

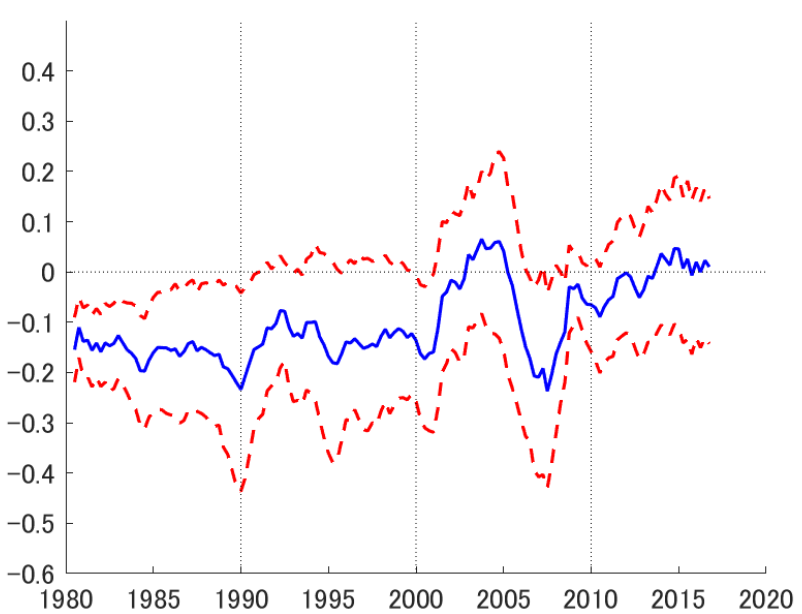

(b) Response after 2nd quarter

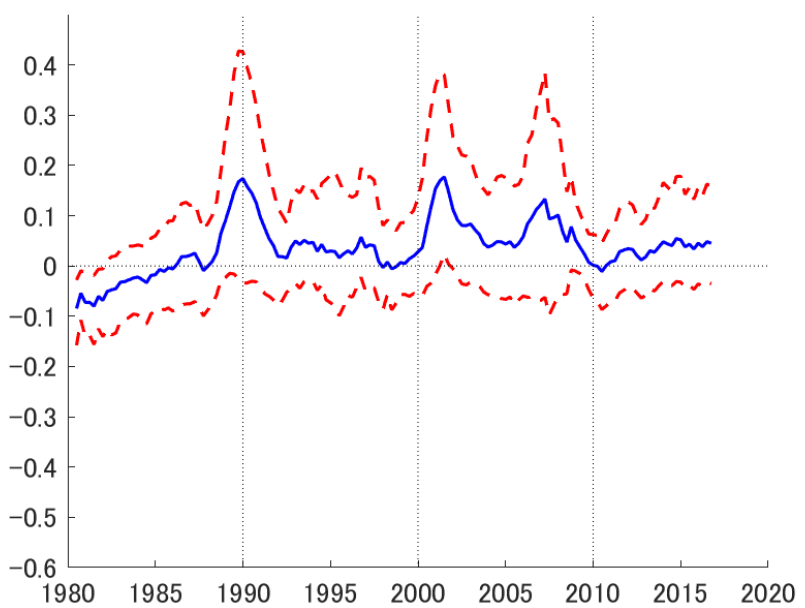

(d) Response after 9th quarter

Notes: Impulse response functions of the fed funds rate (in percent) to a one standard-deviation positive TFP shock. Dotted lines show the $68 \%$ confidence bands estimated by the residual-based block bootstrap method. 
Figure 13: Responses of real interest rate

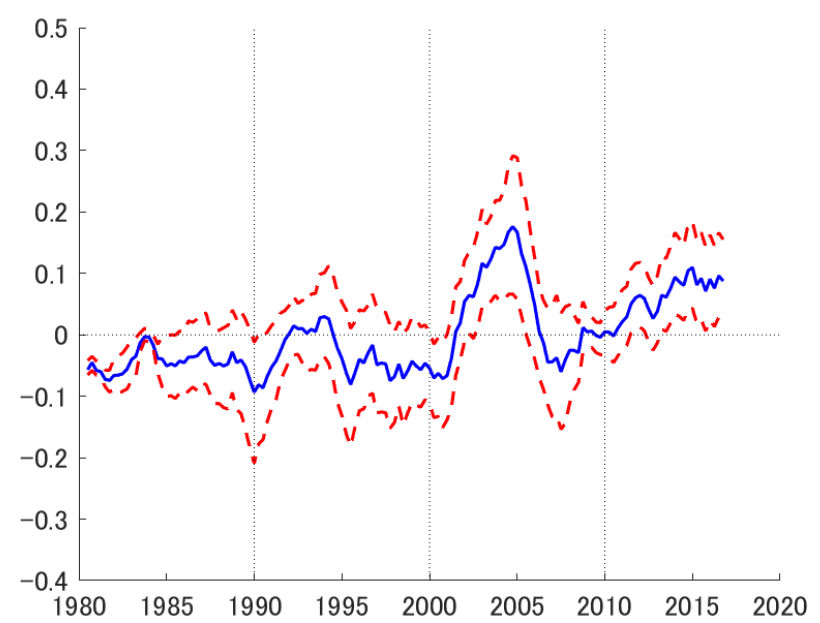

(a) Response after 1st quarter

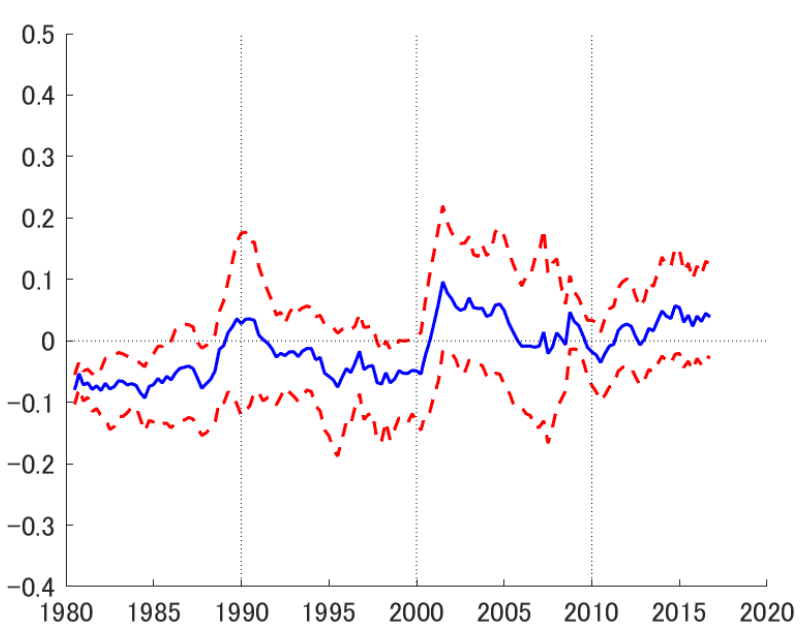

(c) Response after 5th quarter

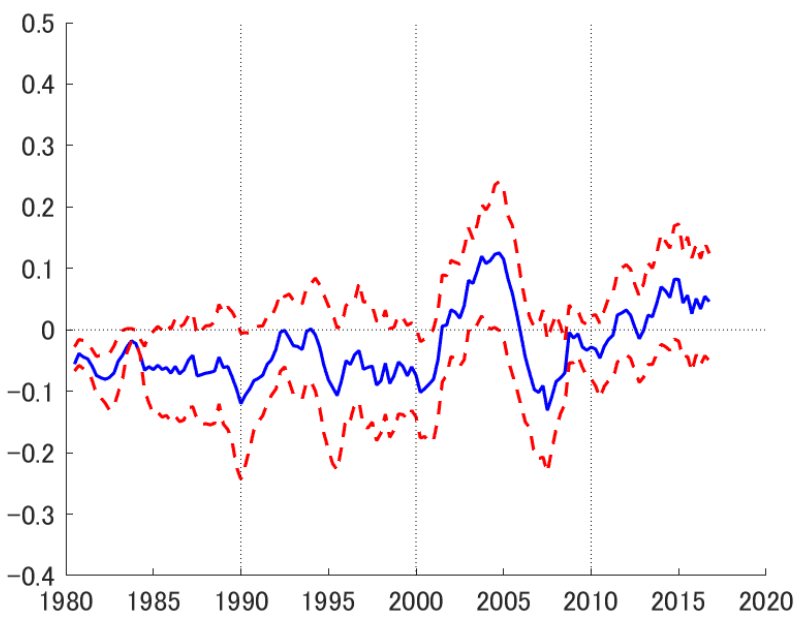

(b) Response after 2nd quarter

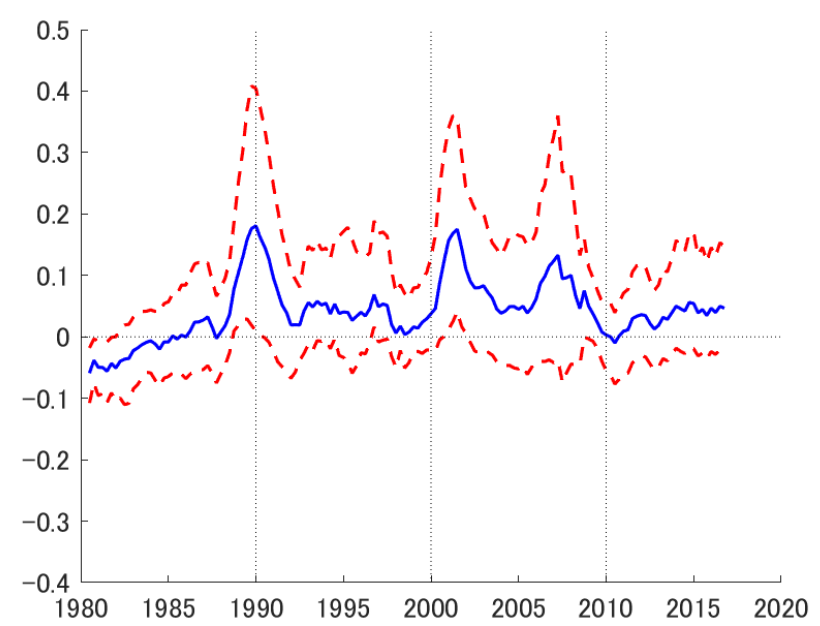

(d) Response after 9th quarter

Notes: Impulse response functions of real interest rate (in percent) to a one standard-deviation positive TFP shock. Dotted lines show the $68 \%$ confidence bands estimated by the residual-based block bootstrap method. 
Figure 14: Responses of cumulative Consumption

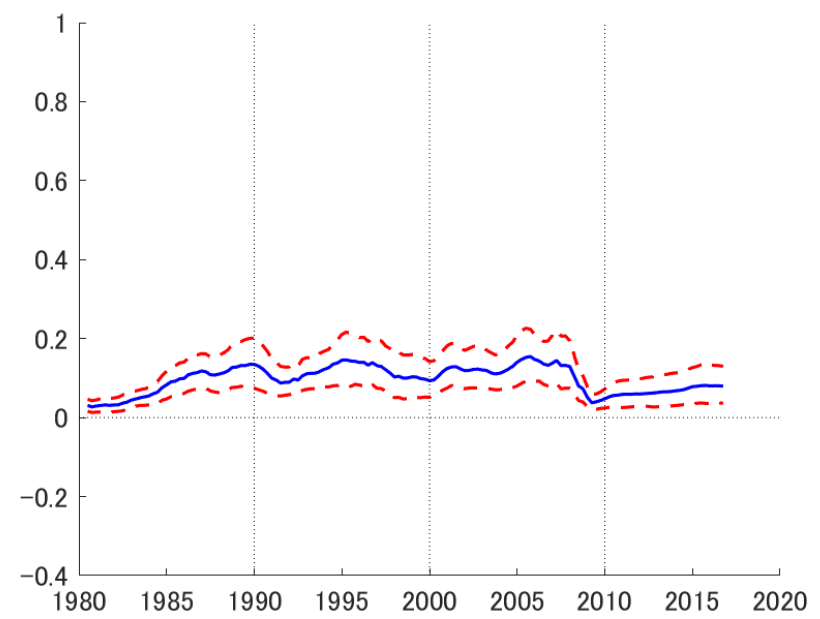

(a) Response after 1st quarter

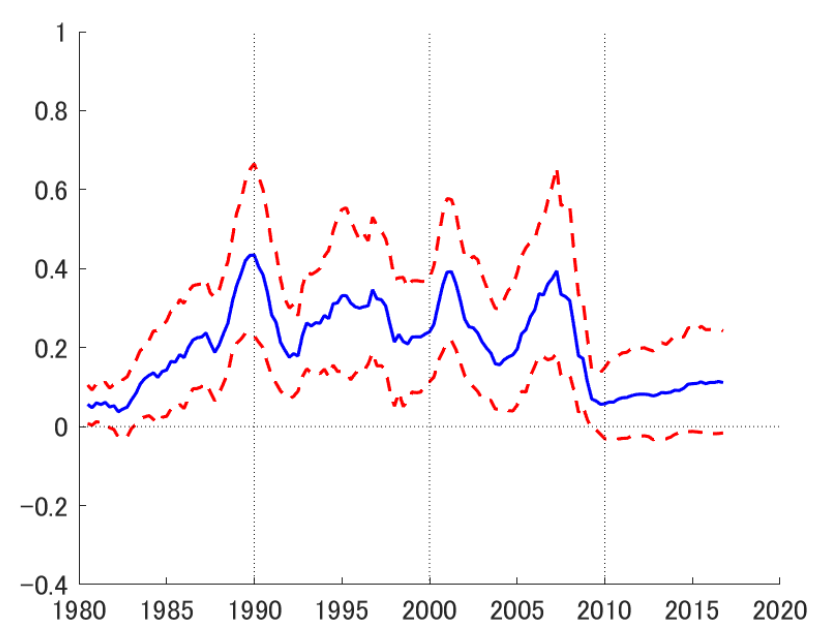

(c) Response after 5th quarter

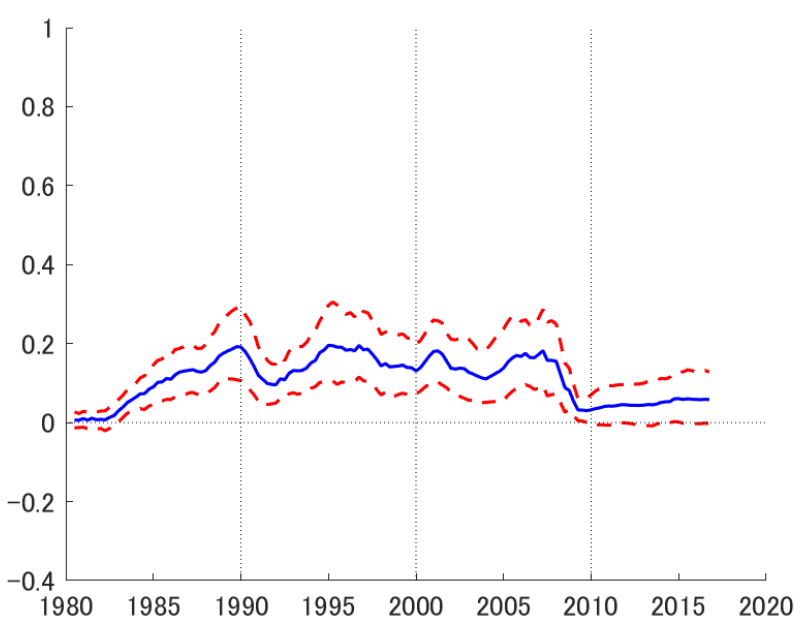

(b) Response after 2nd quarter

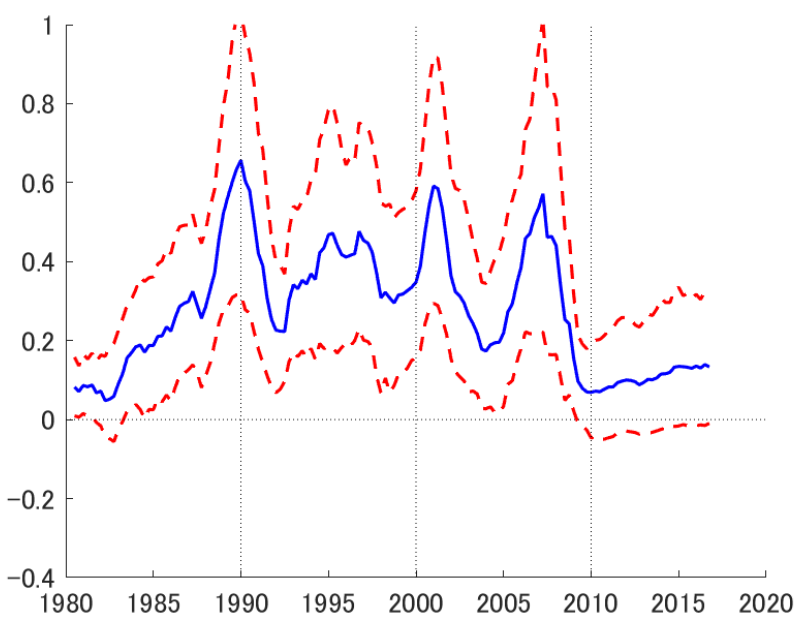

(d) Response after 9th quarter

Notes: Impulse response functions of consumption (in percent) to a one standard-deviation positive TFP shock. Dotted lines show the $68 \%$ confidence bands estimated by the residual-based block bootstrap method. 
Figure 15: Responses of Hours Worked

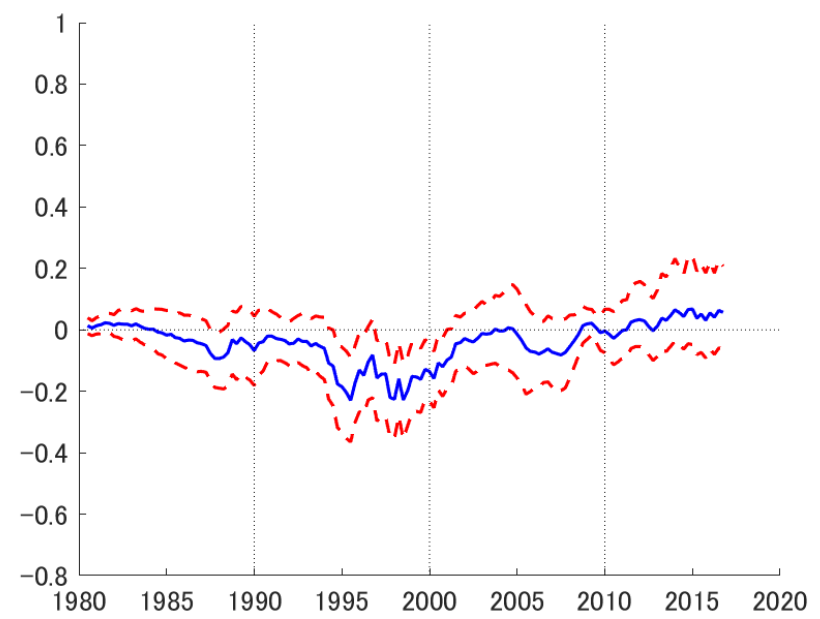

(a) Response after 1st quarter

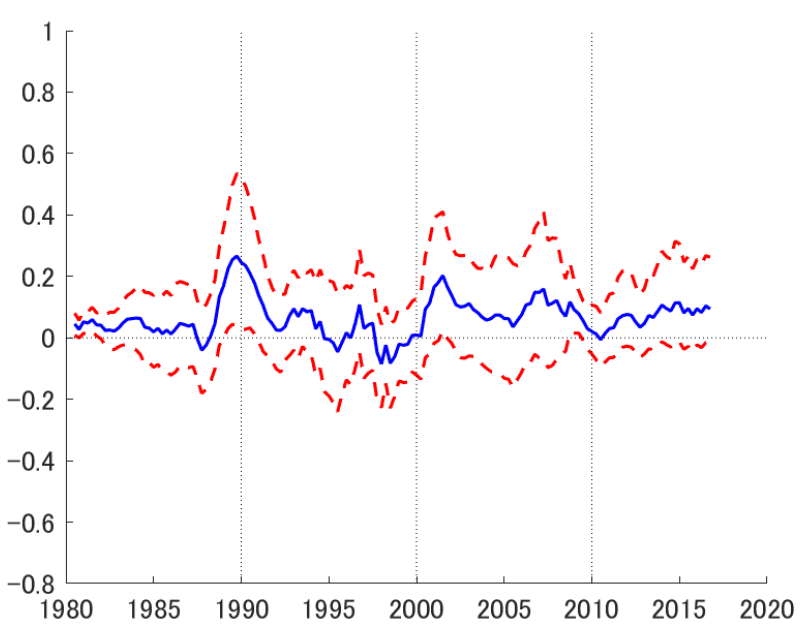

(c) Response after 5th quarter

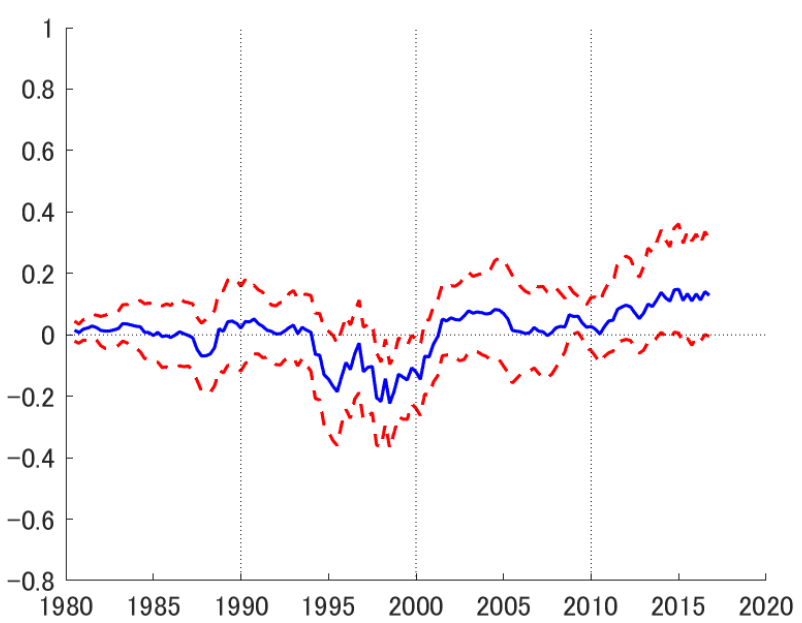

(b) Response after 2nd quarter

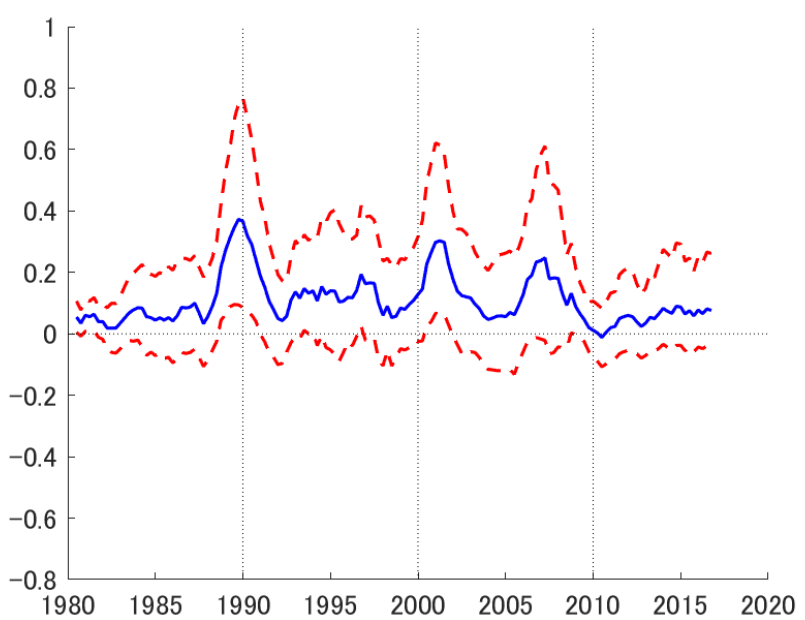

(d) Response after 9th quarter

Notes: Impulse response functions of hours worked (in percent) to a one standard-deviation positive TFP shock. Dotted lines show the $68 \%$ confidence bands estimated by the residual-based block bootstrap method. 
Figure 16: Responses of cumulative Investment

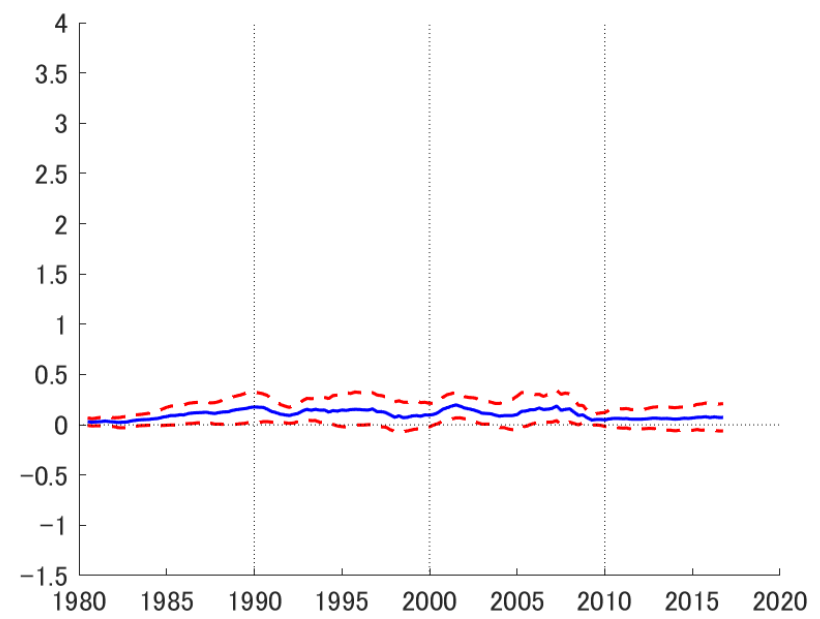

(a) Response after 1st quarter

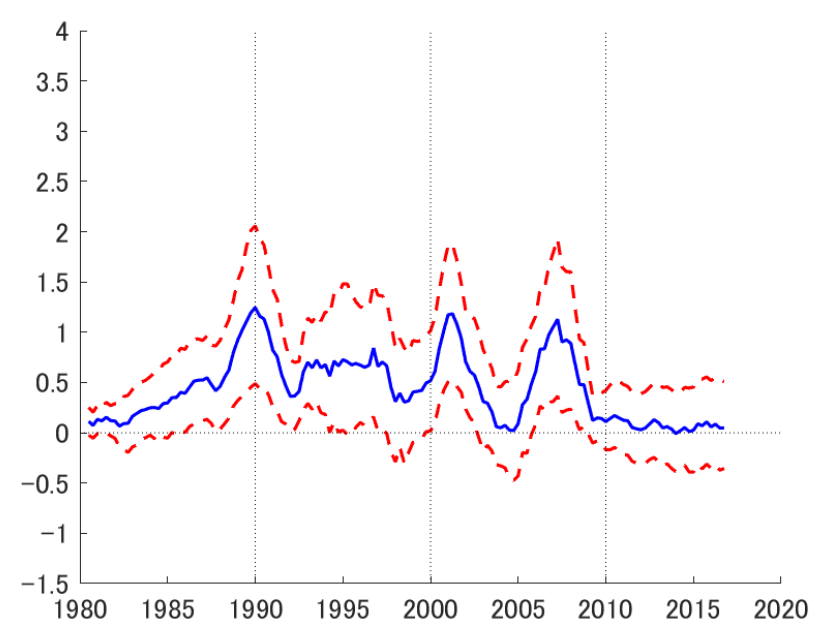

(c) Response after 5th quarter

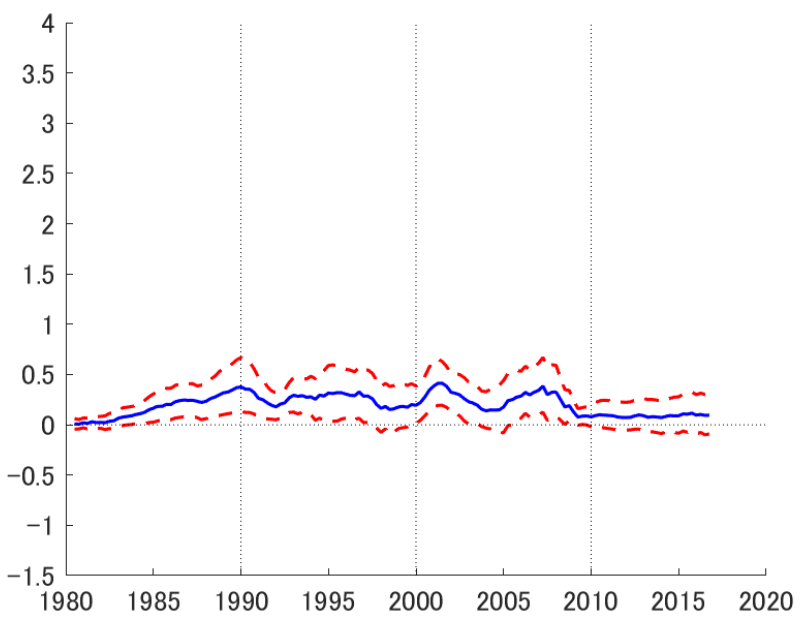

(b) Response after 2nd quarter

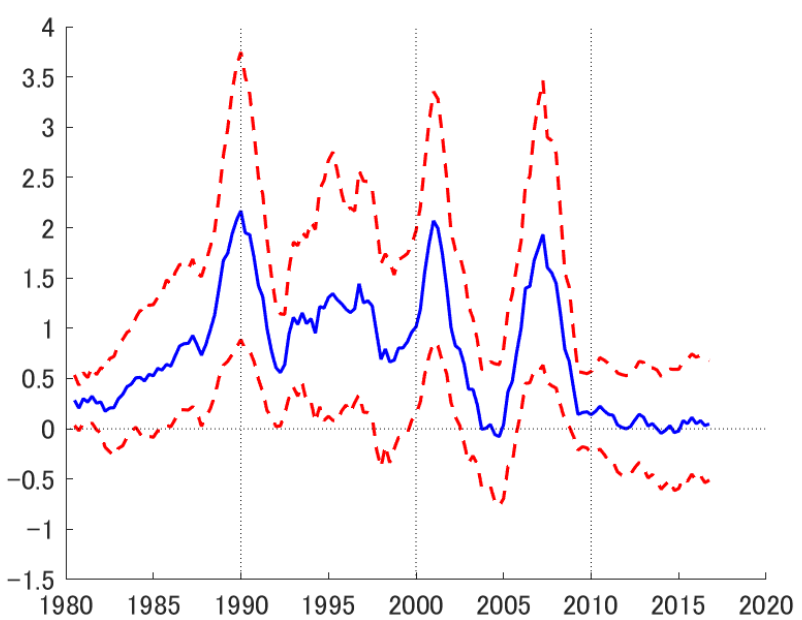

(d) Response after 9th quarter

Notes: Impulse response functions of investment (in percent) to a one standard-deviation positive TFP shock. Dotted lines show the $68 \%$ confidence bands estimated by the residual-based block bootstrap method. 
Figure 17: Response of cumulative GDP using Local Projection method Dependent variable: $\sum_{j=0}^{h} \Delta \ln Y_{t+j}$

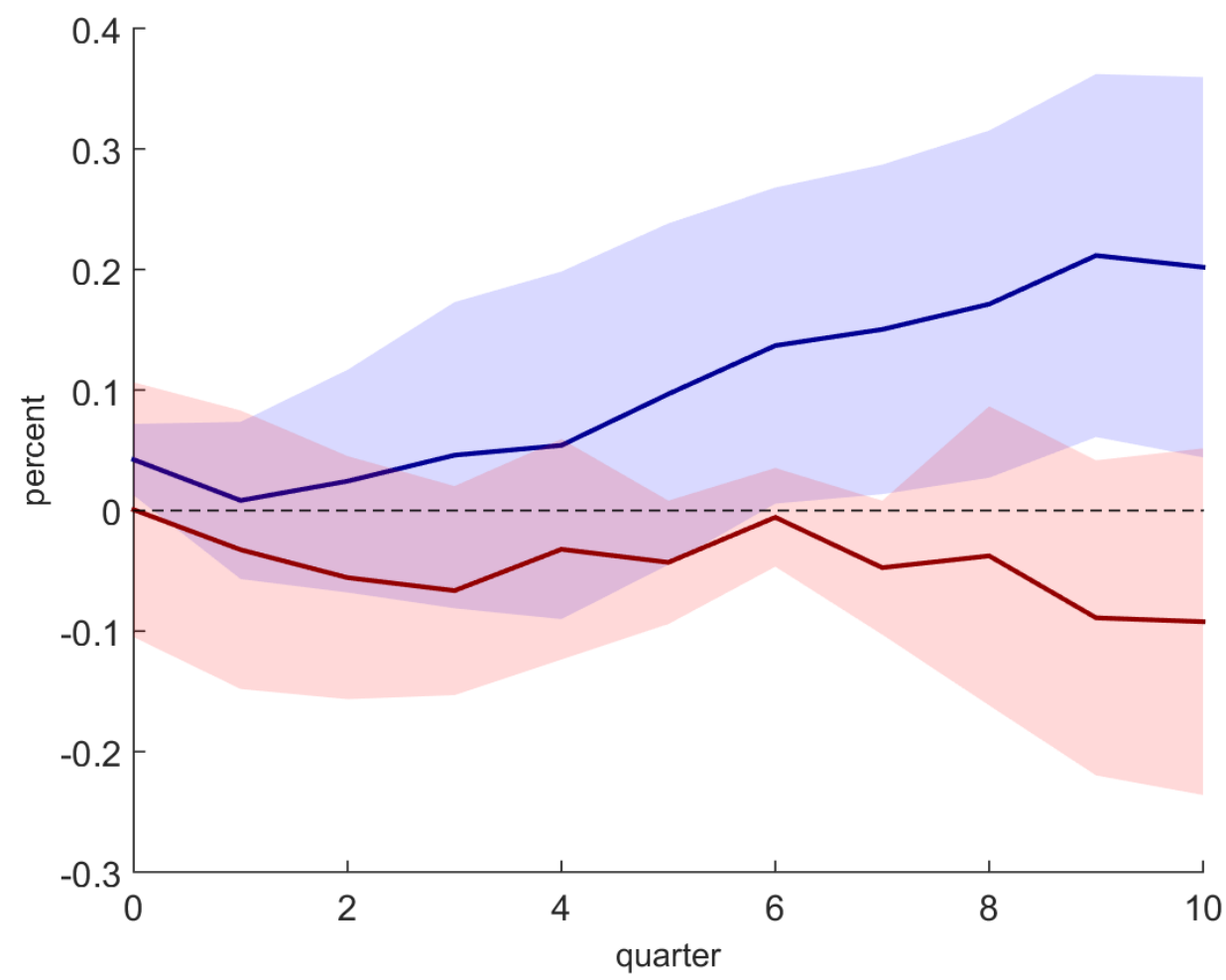

Notes: Estimated impulse response of output to a one unit TFP shock at various horizons. The solid blue line shows the response under the normal periods (i.e. when $\mathrm{Zt}=0$ ). The solid red line is the response when the ZLB binds $(\mathrm{Zt}=1)$. The shaded bands represent the 90 percent confidence interval. 
Figure 18: Response of GDP using Local Projection method

Dependent variable: $\ln Y_{t+h}-\ln Y_{t-1}$

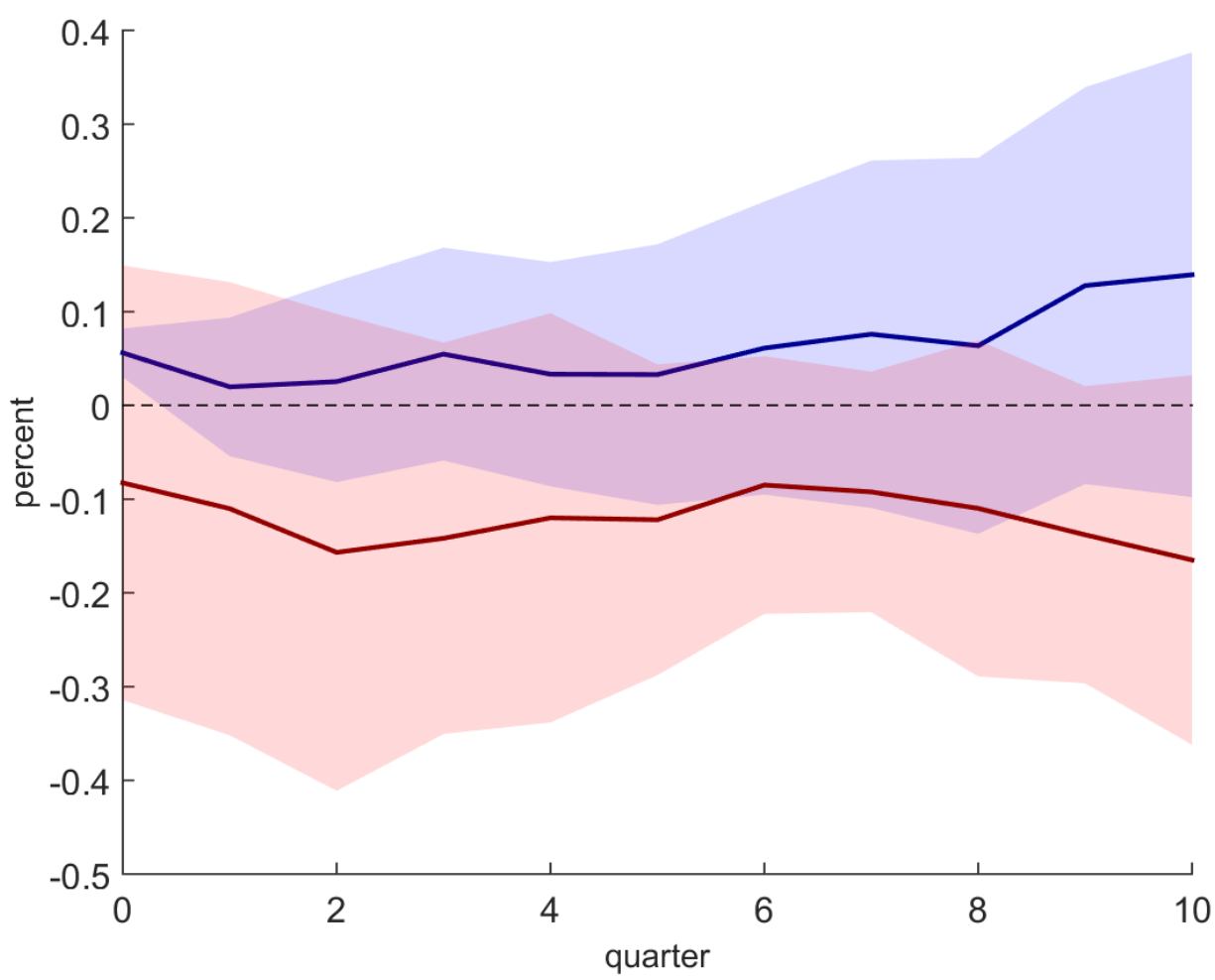

Notes: Estimated impulse response of output to a one unit TFP shock at various horizons. The solid blue line shows the response under the normal periods (i.e. when $\mathrm{Zt}=0$ ). The solid red line is the response when the ZLB binds $(\mathrm{Zt}=1)$. The shaded bands represent the 90 percent confidence interval. 
Figure 19: Residual equation errors

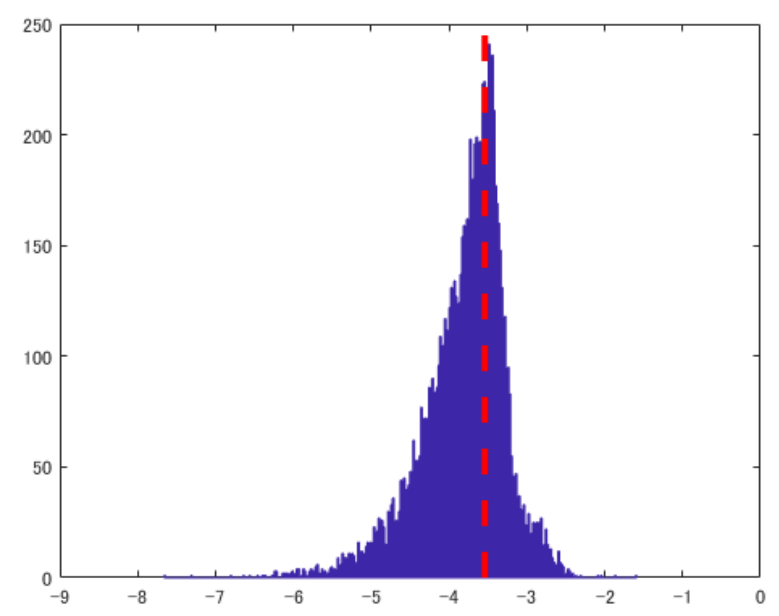

(a) Equation 23

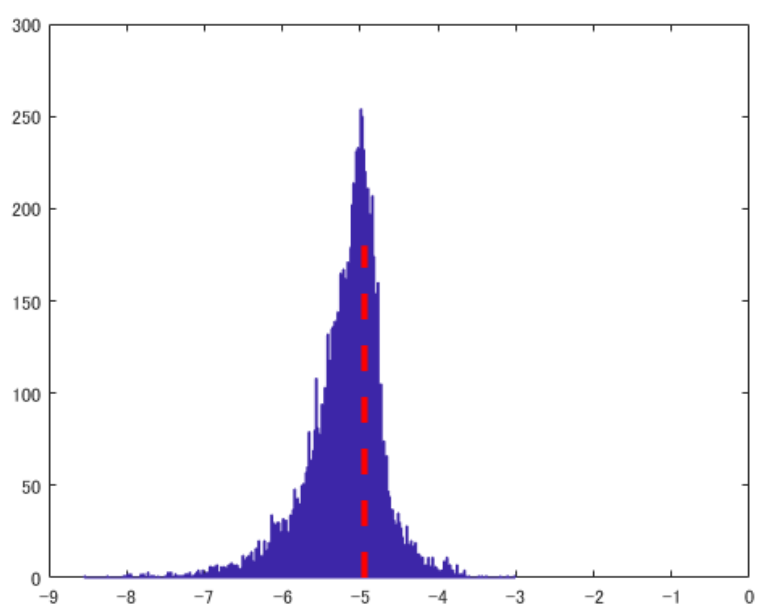

(b) Equation 29

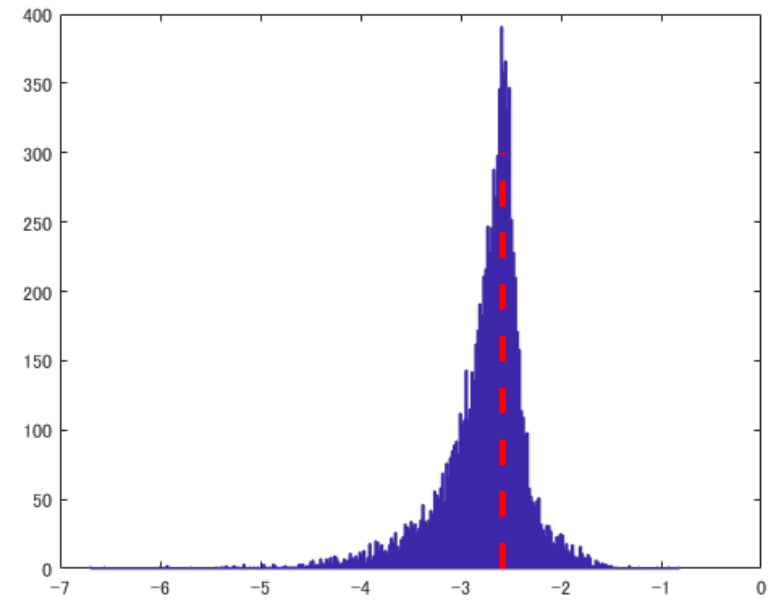

(c) Equation 30

Notes: The histograms report the residual equation errors in decimal log basis. The dotted red lines mark the mean residual equation errors. 